nucl-th/0106067

\title{
Heavy Quarkonium Dissociation Cross Sections in Relativistic Heavy-Ion Collisions
}

\author{
Cheuk-Yin Wong ${ }^{1}$, E. S. Swanson ${ }^{2,3}$ and T. Barnes ${ }^{1,4}$ \\ ${ }^{1}$ Physics Division, Oak Ridge National Laboratory, Oak Ridge, TN 37831 USA \\ ${ }^{2}$ Department of Physics and Astronomy, University of Pittsburgh, Pittsburgh, PA 15260 USA \\ ${ }^{3}$ Jefferson Lab, Newport News, VA 23606 USA \\ ${ }^{4}$ Department of Physics, University of Tennessee, Knoxville, TN 37996 USA
}

\begin{abstract}
Many of the hadron-hadron cross sections required for the study of the dynamics of matter produced in relativistic heavy-ion collisions can be calculated using the quark-interchange model. Here we evaluate the low-energy dissociation cross sections of $J / \psi, \psi^{\prime}, \chi, \Upsilon$, and $\Upsilon^{\prime}$ in collision with $\pi, \rho$, and $K$, which are important for the interpretation of heavy-quarkonium suppression as a signature for the quark gluon plasma. These comover dissociation processes also contribute to heavy-quarkonium suppression, and must be understood and incorporated in simulations of heavy-ion collisions before QGP formation can be established through this signature.
\end{abstract}




\section{INTRODUCTION}

The first collisions of heavy-ion beams at the Relativistic Heavy-Ion Collider (RHIC) at Brookhaven National Laboratory heralded a new era in the study of matter in the extreme conditions of very high temperatures and energy densities. The ultimate goal of this research is the production of a quark-gluon plasma, which is an unusual phase of strongly interacting matter that purportedly existed shortly after the Big Bang [1,2].

The search for the quark-gluon plasma relies on the unusual properties of the plasma for its detection. However, many conventional hadrons are also produced during a heavy ion collisions. Whatever signal is chosen for the identification of the quark-gluon plasma, contributions to that signal from conventional hadronic processes must be identified as backgrounds and removed from the data.

Although there are recent, tantalizing hints of possible quark-gluon plasma production in heavy-ion collisions at CERN [3,4], conclusive evidence is still lacking due to uncertain backgrounds from conventional hadronic sources. Investigations of the various 'hadronic background processes' are urgently needed if we are to develop a satisfactory understanding of the various signals proposed as signatures of a quark-gluon plasma. In this paper we consider one type of hadronic background processes that can contribute to heavy-quarkonium suppression, which is frequently cited as a QGP signature.

Matsui and Satz [5] originally suggested the use of suppressed $J / \psi$ production as a signature for the formation of a quark-gluon plasma in high-energy heavy-ion collisions. The recent experimental observation of anomalous $J / \psi$ suppression in $\mathrm{Pb}+\mathrm{Pb}$ collisions by the NA50 Collaboration [6,7] has been considered by many authors 8 17]. However there is considerable uncertainty in these studies, due to the lack of reliable experimental information on $J / \psi$ and $\chi_{J}$ dissociation cross sections in low-energy collisions with light hadrons. Because heavy quarkonia decay strongly, many of the dissociation cross sections cannot be measured directly in hadron scattering experiments; the cross sections are instead typically estimated using theoretical models. Evaluation of these cross sections is of particular interest for clarifying the physics of the $J / \psi$ anomalies observed in $\mathrm{Pb}-\mathrm{Pb}$ collisions, and may be of considerable importance in future $J / \psi$ studies using the RHIC and LHC colliders.

The dissociation of the $J / \psi$ by hadrons has been considered previously in several theoretical studies, but the predicted cross sections show great variation at low energies, largely due to different assumptions regarding the dominant scattering mechanism [18 28].

Kharzeev, Satz, and collaborators [18, 19] employed the parton model and perturbative QCD "short-distance" approach of Bhanot and Peskin [29,30, and found remarkably small low-energy cross sections for collisions of $J / \psi$ with light hadrons. For example, their $J / \psi+N$ cross section at $\sqrt{s}=5 \mathrm{GeV}$ is only about $0.25 \mu \mathrm{b}$ [18]. A finite-mass correction increases this cross section by about a factor of two [19]. However, in high-energy heavy-ion reactions the collisions between the produced $\pi$ and $\rho$ with $J / \psi$ and $\psi^{\prime}$ occur at low energies (typically from a few hundred $\mathrm{MeV}$ to about $1 \mathrm{GeV}$ relative kinetic energies). The applicability of the parton model and pQCD for reactions in this low-energy region is certainly open to question.

Matinyan and Müller [24], Haglin [25], Lin and Ko [26], and Oh, Song, and Lee [27] recently reported results for these dissociation cross sections in meson exchange models. These references all use effective meson Lagrangians, but differ in the interaction terms included in the Lagrangian. Matinyan and Müller included only $t$-channel $D$ meson exchange, and 
found that the dissociation cross sections of $J / \psi$ by $\pi$ and $\rho$ are rather small; both are $\approx 0.2-0.3 \mathrm{mb}$ at $\sqrt{s}=4 \mathrm{GeV}$. Including form factors (arbitrarily chosen to be Gaussians with a width set to $1.5 \mathrm{GeV}$ ) would reduce these cross sections by an order of magnitude. Haglin obtained a very different result, with much larger cross sections, by treating the $D$ and $D^{*}$ mesons as non-Abelian gauge bosons in a minimally coupled Yang-Mills meson Lagrangian. Form factors were also introduced in these calculations [25 27]. The resulting mb-scale cross sections are very sensitive to the choice of form factors. Charmonium dissociation by nucleons has also been considered recently using a similar effective Lagrangian formulation [28]. Of course the use of a Yang-Mills Lagrangian for charmed mesons has no a priori justification, so this crucial initial assumption made in these references requires independent confirmation. The assumption of the $t$-channel exchange of a heavy meson such as a $D$ or $D^{*}$ between a hadron and a $J / \psi$ is also difficult to justify physically, because the range of these exchanges $(1 / M \approx 0.1 \mathrm{fm})$ is much smaller than the physical extent of the initial hadron and the $J / \psi$.

Charmonium dissociation processes can undoubtedly be described in terms of interquark interactions, as we attempt in this paper. Since these reactions are of greatest phenomenological interest at energy scales in the resonance region, we advocate the use of the known quark forces to obtain the underlying scattering amplitudes from explicit nonrelativistic quark model hadron wavefunctions of the initial and final mesons.

Martins, Blaschke, and Quack [20] previously reported dissociation cross section calculations using essentially the same approach we describe here. The short-distance interaction used by these authors in particular is quite similar to the form we employ. For the confining interaction, however, they used a simplified color-independent Gaussian potential between quark-antiquark pairs, rather than the now well-established linear $\boldsymbol{\lambda}(i) \cdot \boldsymbol{\lambda}(j)$ form. They found a rather large $\pi+J / \psi$ dissociation cross section, which reached a maximum of about $7 \mathrm{mb}$ at a center-of-mass kinetic energy $E_{K E}$ of about $0.85 \mathrm{GeV}$. Although our approach is very similar to that of Martins et al., our final numerical results differ significantly, due mainly to our different treatments of the confining interaction.

In this paper we use the approach discussed above to evaluate the dissociation cross sections of $J / \psi, \psi^{\prime}, \chi, \Upsilon$, and $\Upsilon$ by $\pi, \rho$, and $K$, and compare our results to other theoretical cross sections reported in the literature. The dissociation cross sections of $\chi_{J}$ mesons are of special interest, as about $1 / 3$ of the $J / \psi$ mesons produced in a high-energy nucleon-nucleon collision come from the decay of $\chi$ states [31]. The dissociation cross sections for $\Upsilon$ are also interesting and they have recently been estimated to be quite small in an effectiveLagrangian meson exchange model because of large thresholds for the dissociation of $\Upsilon$ by both $\pi$ and $\rho$ [32].

We employ the Barnes-Swanson quark-interchange model [33, 34 to evaluate these dissociation cross sections in terms of wavefunctions and interactions at the quark level. We use the nonrelativistic quark potential model and its interquark Hamiltonian to describe the underlying quark-gluon forces. The model parameters and quark masses are determined by the meson spectrum, so there is little additional freedom in determining scattering amplitudes and cross sections. We thus implicitly incorporate the successes of the quark model in describing the meson spectrum and many static and dynamical properties of hadrons. We proceed by calculating the scattering amplitude for a given process at Born order in the interquark Hamiltonian; the good agreement of this approach with experimental scattering 
data on many low-energy reactions 33 36] provides strong motivation for the application of this approach to hadron reactions in relativistic heavy-ion collisions. A brief summary of the present work has been reported previously [21].

This paper is organized as follows. In Section II we summarize the Barnes-Swanson model of quark interchange as applied to the calculations of the dissociation cross sections. The reaction matrix can be described in terms of the "prior" or "post" diagrams, which are discussed in Section III. Section IV gives some details of the evaluation of the spin and spatial matrix elements for the general meson-meson scattering problem. In Section V the spin matrix elements are derived explicitly in terms of 9- $j$ symbols. The evaluation of spatial overlap integrals for the case of all $S$-wave mesons is discussed in Section VI. In Section VII, we evaluate the corresponding overlap integrals for one $P$-wave meson. An accurate determination of these matrix elements requires correspondingly accurate bound state wavefunctions; the evaluation of these wavefunctions is discussed in Section VIII. The numerical agreement between the post and prior scattering formalisms is demonstrated explicitly in Section IX, which provides a very nontrivial check of the accuracy and internal consistency of our calculations. Section X presents our results for the dissociation cross section of $J / \psi$ and $\psi^{\prime}$ in collision with various light mesons, and Section XI gives the corresponding cross sections for the dissociation of $\Upsilon$ and $\Upsilon^{\prime}$. Section XII present results for the dissociation of $P$-wave charmonium states, the $\chi_{J}$ mesons, in collision with $\pi, \rho$, and $K$. Finally, we present conclusions in Section XIII.

\section{THE MODEL}

We shall briefly summarize the model of Barnes and Swanson for constituent-interchange processes in the reaction

$$
A(12)+B(34) \rightarrow C(14)+D(32)
$$

where $A, B, C$, and $D$ are $q \bar{q}$ mesons, and 1, 3, and 2, 4 label the quark and antiquark constituents respectively. In this meson-meson scattering problem the scattering amplitude in the "prior formalism" is the sum of the four quark-line diagrams of Fig. 1. These are evaluated as overlap integrals of quark model wavefunctions using the "Feynman rules" given in App. C of Ref. [33]. This method has previously been applied successfully to the closely related no-annihilation scattering channels $I=2 \pi \pi[33], I=3 / 2 K \pi$ [35], $I=\{0,1\}$ $S$-wave $K N$ scattering [36], and the short-range repulsive $N N$ interaction [37].

The interaction $H_{i j}\left(r_{i j}\right)$ between the pair of constituents $i$ and $j$ is represented by the curly line in Fig. 1 and is taken to be

$$
\begin{aligned}
H_{i j}\left(r_{i j}\right) & =\frac{\boldsymbol{\lambda}(i)}{2} \cdot \frac{\boldsymbol{\lambda}(j)}{2}\left\{V_{\text {color-Coulomb }}\left(r_{i j}\right)+V_{\text {linear }}\left(r_{i j}\right)+V_{\text {spin-spin }}\left(r_{i j}\right)+V_{\text {con }}\right\} \\
& =\frac{\boldsymbol{\lambda}(i)}{2} \cdot \frac{\boldsymbol{\lambda}(j)}{2}\left\{\frac{\alpha_{s}}{r_{i j}}-\frac{3 b}{4} r_{i j}-\frac{8 \pi \alpha_{s}}{3 m_{i} m_{j}} \boldsymbol{s}_{i} \cdot \boldsymbol{s}_{j}\left(\frac{\sigma^{3}}{\pi^{3 / 2}}\right) e^{-\sigma^{2} r_{i j}^{2}}+V_{\text {con }}\right\},
\end{aligned}
$$

where $\alpha_{s}$ is the strong coupling constant, $b$ is the string tension, $m_{i}$ and $m_{j}$ are the masses of the interacting constituents, $\sigma$ is the range parameter in the hyperfine spin-spin interaction, 
and $V_{\text {con }}$ is a constant. For an antiquark, the generator $\boldsymbol{\lambda} / 2$ is replaced by $-\boldsymbol{\lambda}^{T} / 2$.
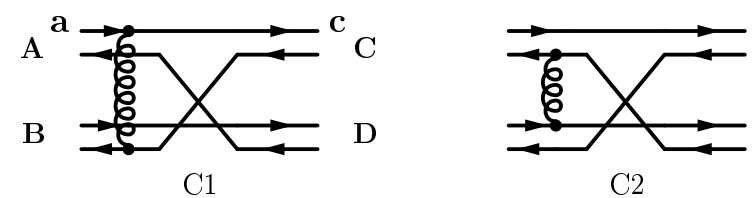

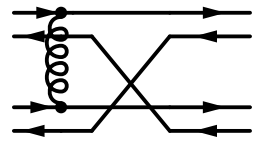

$\mathrm{T} 1$

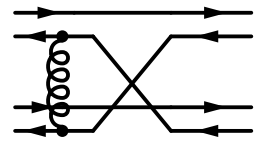

$\mathrm{T} 2$

Fig. 1. 'Prior' diagrams for Born-order meson-meson scattering.

It is convenient to introduce $V_{i j}\left(r_{i j}\right)$ to denote the quantity in curly brackets in Eq. (2) so that we can write $H_{i j}\left(\boldsymbol{r}_{i j}\right)$ in the form

$$
H_{i j}\left(\boldsymbol{r}_{i j}\right)=\frac{\boldsymbol{\lambda}(i)}{2} \cdot \frac{\boldsymbol{\lambda}(j)}{2} V_{i j}\left(r_{i j}\right)
$$

The Born-order $T$-matrix element $T_{f i}$ is proportional to the matrix elements $h_{f i}$ of this residual interaction (as defined in Ref. [33]). For each of the scattering diagrams of Fig. 1, $h_{i j}$ and $T_{i j}$ are given as the product of four factors,

$$
h_{f i}=\frac{1}{(2 \pi)^{3}} T_{f i}=S I_{\text {flavor }} I_{\text {color }} I_{\text {spin-space }} \text {. }
$$

The overall sign $S$ is a fermion-permutation phase known as the "signature" of the diagram, which is equal to $(-1)^{N_{x}}$, where $N_{x}$ is the number of fermion line crossings. $(S=-1$ for the diagrams in Fig. 1.) The flavor matrix element $I_{\text {flavor }}$ is the overlap of the initial and final flavor wavefunctions. In all the processes considered in this paper, $I_{\text {flavor }}$ is equal to 1 for all diagrams. The color matrix element $I_{\text {color }}$ is $-4 / 9$ for diagrams C1 and C2 of Fig. 1, and is $+4 / 9$ for diagrams T1 and T2. The spatial and spin matrix element $I_{\text {spin-space }}$ is the matrix element of $V_{i j}$, and can in general be written as a sum of products of a spin matrix element $I_{\text {spin }}$ times a spatial matrix elements $I_{\text {space }}$. The spin matrix element $I_{\text {spin }}$ involves Clebsch-Gordon coefficients and the spins of the colliding particles and is tabulated for all cases of $S$-wave mesons in 33. An explicit closed-form expression for this $I_{\text {spin }}$ in terms of Wigner's $9 j$ symbols will be given in Section V. The evaluation of the spatial matrix element $I_{\text {space }}$ will be discussed in detail in Sections VI and VII.

For the reaction $A+B \rightarrow C+D$, with an invariant momentum transfer $t$

$$
t=(A-C)^{2}=m_{A}^{2}+m_{C}^{2}-2 A_{0} C_{0}+2 \boldsymbol{A} \cdot \boldsymbol{C},
$$

the differential cross section is given by

$$
\frac{d \sigma_{f i}}{d t}=\frac{1}{64 \pi s\left|\boldsymbol{p}_{A}\right|^{2}}\left|\mathcal{M}_{f i}\right|^{2}
$$

where the matrix element $\mathcal{M}_{f i}$ is related to $T_{f i}$ by 


$$
\mathcal{M}_{f i}=\sqrt{\left(2 E_{A}\right)\left(2 E_{B}\right)\left(2 E_{C}\right)\left(2 E_{D}\right)} T_{f i} .
$$

In Eqs. (6) and (7), $\boldsymbol{p}_{A}$ and $E_{A}$ are the momentum and the energy of meson $A$ in the center-of-mass system. The total cross section for the reaction $A+B \rightarrow C+D$ can be obtained from $d \sigma_{f i} / d t$ by integrating over $t$.

\section{POST AND PRIOR DESCRIPTIONS}

Before proceeding to our results, we note that a well-known "post-prior" ambiguity arises in the calculation of bound state scattering amplitudes involving rearrangement collisions [39]. Since the Hamiltonian which describes the scattering process $A B \rightarrow C D$ can be separated into an unperturbed Hamiltonian and a residual interaction in two ways, $H=$ $H_{A}^{(0)}+H_{B}^{(0)}+V_{A B}=H_{C}^{(0)}+H_{D}^{(0)}+V_{C D}$, there is an ambiguity in the choice of $V_{A B}$ or $V_{C D}$ as the residual interaction. The first version gives the "prior" diagrams of Fig. 1, in which the interaction occurs before constituent interchange. The second choice is the "post" formalism in which the interaction occurs after constituent interchange, as in the diagrams of Fig. 2.
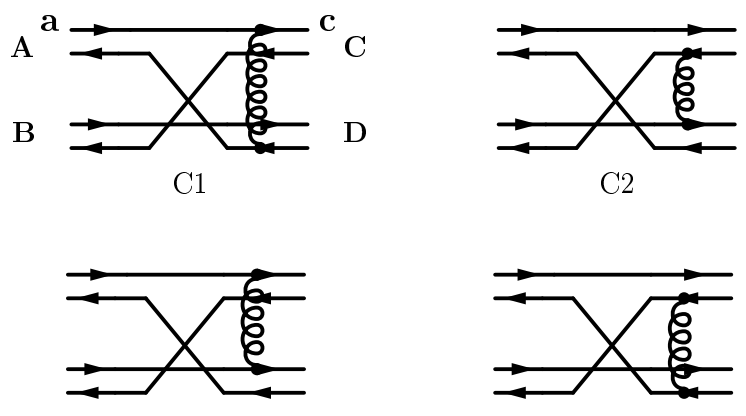

$\mathrm{T} 1$

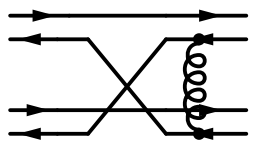

$\mathrm{T} 2$

Fig. 2. 'Post' diagrams for Born-order meson-meson scattering.

One may prove in the context of non-relativistic quantum mechanics that the 'prior' and 'post' diagrams give the same scattering amplitude and hence the same cross section, provided that exact bound state wavefunctions of the various $\left\{H^{(0)}\right\}$ are used for the external states [39]. (This is discussed in detail and is demonstrated numerically in Ref. [34] for $\pi \pi \rightarrow \rho \rho$ scattering.) A consistent calculation thus leads to description-identical results for the scattering amplitude in non-relativistic quantum mechanics. We shall confirm the "prior-post" equivalence numerically in our non-relativistic calculations of the $J / \psi$ and $\psi^{\prime}$ dissociation cross sections.

\section{EVALUATION OF THE MATRIX ELEMENT $I_{\text {spin-space }}$}

For the processes considered here, it suffices to treat reactions of the form $A(12)+$ $B(34) \rightarrow C(14)+D(32)$, in which constituents (antiquarks) 2 and 4 are interchanged, as depicted in Figs. 1 and 2. We denote the total angular momentum, the orbital angular momentum, and the spin of meson $a(a=A, B, C$, and $D)$ by $J_{a}, L_{a}$, and $S_{a}$ respectively, with the associated spatial wavefunction $\Phi_{a}$ and spin wavefunction $\chi_{a}$. 
The quantity $I_{\text {spin-space }}$ is the matrix element of $V_{i j}\left(r_{i j}\right)$ between the initial and final meson states. The interaction $V_{i j}\left(r_{i j}\right)$ is the spin and spatial part of $H_{i j}$ (Eqs. (21) and (3)), and it consists of the sum

$$
V_{i j}\left(r_{i j}\right)=\sum_{i}^{3} v_{r}^{(i)} v_{s}^{(i)}+V_{\text {con }}
$$

where the superscripts $(i)$ represent the color-Coulomb, linear, and spin-spin interactions respectively. Specifically, $v_{s}^{(1)}=v_{s}^{(2)}=1, v_{s}^{(3)}=\boldsymbol{s}_{i} \cdot \boldsymbol{s}_{j}$, and the corresponding $v_{r}^{(i)}$ can be determined from Eqs. (21) and (3). For the scattering problem the sum of the amplitudes of all diagrams arising from the constant term $V_{\text {con }}$ is zero, so we do not need to include $V_{\text {con }}$ in deriving scattering amplitudes and matrix elements.

The matrix element $I_{\text {spin-space }}$ is therefore the sum of three terms, each of which is of the form

$$
\begin{aligned}
& \left\langle\left(\Phi_{C} \chi_{C}\right)_{J_{C z}}^{J_{C}}\left(\Phi_{D} \chi_{D}\right)_{J_{D z}}^{J_{D}}\left|v_{r} v_{s}\right|\left(\Phi_{A} \chi_{A}\right)_{J_{A z}}^{J_{A}}\left(\Phi_{B} \chi_{B}\right)_{J_{B z}}^{J_{B}}\right\rangle \\
& =\sum_{J J_{z} J^{\prime} J_{z}^{\prime}}\left(J_{C} J_{C z} J_{D} J_{D z} \mid J^{\prime} J_{z}^{\prime}\right)\left(J J_{z} \mid J_{A} J_{A z} J_{B} J_{B z}\right) \\
& \quad \times\left\langle\left[\left(\Phi_{C} \chi_{C}\right)^{J_{C}}\left(\Phi_{D} \chi_{D}\right)^{J_{D}}\right]_{J_{z}^{\prime}}^{J^{\prime}}\left|v_{r} v_{s}\right|\left[\left(\Phi_{A} \chi_{B}\right)^{J_{A}}\left(\Phi_{B} \chi_{B}\right)^{J_{B}}\right]_{J_{z}}^{J}\right\rangle .
\end{aligned}
$$

In the above equation, the coupled initial state $\left|\left[\left(\Phi_{A} \chi_{B}\right)^{J_{A}}\left(\Phi_{B} \chi_{B}\right)^{J_{B}}\right]_{J_{z}}^{J}\right\rangle \equiv\left|\Psi_{\mathrm{in}}^{J J_{z}}\right\rangle$ of mesons $A(12)$ and $B(34)$ can be written as 40]

$$
\begin{aligned}
\left|\Psi_{\mathrm{in}}^{J J_{z}}\right\rangle & =\sum_{S, L}\left\langle\left(\chi_{A} \chi_{B}\right)^{S}\left(\Phi_{A} \Phi_{B}\right)^{L} J J_{z} \mid\left(\Phi_{A} \chi_{B}\right)^{J_{A}}\left(\Phi_{B} \chi_{B}\right)^{J_{B}} J J_{z}\right\rangle\left|\left[\left(\chi_{A} \chi_{B}\right)^{S}\left(\Phi_{A} \Phi_{B}\right)^{L}\right]_{J_{z}}^{J}\right\rangle \\
& =\sum_{S L S_{z}} \hat{S} \hat{L} \hat{J}_{A} \hat{J}_{B}\left\{\begin{array}{ccc}
S_{A} & S_{B} & S \\
L_{A} & L_{B} & L \\
J_{A} & J_{B} & J
\end{array}\right\}\left(S S_{z} L\left(J_{z}-S_{z}\right) \mid J J_{z}\right)\left|\left(\chi_{A} \chi_{B}\right)_{S_{z}}^{S}\left(\Phi_{A} \Phi_{B}\right)_{\left(J_{z}-S_{z}\right)}^{L}\right\rangle,
\end{aligned}
$$

where $\hat{S} \equiv \sqrt{2 S+1}$. The final state $\left|\left[\left(\Phi_{C} \chi_{C}\right)^{J_{C}}\left(\Phi_{D} \chi_{D}\right)^{J_{D}}\right]_{J_{z}^{J^{\prime}}}^{J^{\prime}}\right\rangle \equiv\left|\Psi_{\text {final }}^{J^{\prime} J_{z}^{\prime}}\right\rangle$ of mesons $C(14)$ and $D(32)$ can be written in a similar way, so the matrix element on the righthand side of Eq. (9) is

$$
\begin{aligned}
\left\langle\Psi_{\text {final }}^{J^{\prime} J_{z}^{\prime}}\left|v_{r} v_{s}\right| \Psi_{\text {in }}^{J J_{z}}\right\rangle & =\sum_{S L S_{z} S^{\prime} L^{\prime} S_{z}^{\prime}} \hat{S} \hat{L} \hat{J}_{A} \hat{J}_{B}\left\{\begin{array}{ccc}
S_{A} & S_{B} & S \\
L_{A} & L_{B} & L \\
J_{A} & J_{B} & J
\end{array}\right\} \hat{S}^{\prime} \hat{L}^{\prime} \hat{J}_{C} \hat{J}_{D}\left\{\begin{array}{ccc}
S_{C} & S_{D} & S^{\prime} \\
L_{C} & L_{D} & L^{\prime} \\
J_{C} & J_{D} & J^{\prime}
\end{array}\right\} \\
& \times\left(S S_{z} L\left(J_{z}-S_{z}\right) \mid J J_{z}\right)\left(S^{\prime} S_{z}^{\prime} L^{\prime}\left(J_{z}^{\prime}-S_{z}^{\prime}\right) \mid J^{\prime} J_{z}^{\prime}\right)^{*} \\
& \times\left\langle\left(\Phi_{C} \Phi_{D}\right)_{\left(J_{z}^{\prime}-S_{z}^{\prime}\right)}^{L^{\prime}}\left|v_{r}\right|\left(\Phi_{A} \Phi_{B}\right)_{\left(J_{z}-S_{z}\right)}^{L}\right\rangle\left\langle\left(\chi_{C} \chi_{D}\right) S_{S_{z}^{\prime}}^{S^{\prime}}\left|v_{s}\right|\left(\chi_{A} \chi_{B}\right)_{S_{z}}^{S}\right\rangle .
\end{aligned}
$$

The above result shows that $I_{\text {spin-space }}$ is in general a sum of products of a spatial matrix element $\left\langle\left(\Phi_{C} \Phi_{D}\right)_{\left(J_{z}^{\prime}-S_{z}^{\prime}\right)}^{L^{\prime}}\left|v_{r}\right|\left(\Phi_{A} \Phi_{B}\right)_{\left(J_{z}-S_{z}\right)}^{L}\right\rangle$ and a spin matrix element $I_{\text {spin }}=\left\langle\left(\chi_{C} \chi_{D}\right)_{S_{z}^{\prime}}^{S^{\prime}}\left|v_{s}\right|\left(\chi_{A} \chi_{B}\right)_{S_{z}}^{S}\right\rangle$. For our interaction, the spin matrix element $\left\langle\left(\chi_{C} \chi_{D}\right) S_{S_{z}^{\prime}}^{S^{\prime}}\left|v_{s}\right|\left(\chi_{A} \chi_{B}\right)_{S_{z}}^{S}\right\rangle$ is diagonal in $S$ and $S_{z}$, and is independent of $S_{z}$, as shown in the next section. 
In this paper, we shall specialize to the cases in which mesons $B, C$ and $D$ are all $S$-wave mesons with $L_{B}=L_{C}=L_{D}=0$. Therefore we have $L=L_{A}, J_{B}=S_{B}, J^{\prime}=S, J_{z}^{\prime}=S_{z}$, and

$$
\begin{aligned}
\left\langle\Psi_{\text {final }}^{S S_{z}}\left|v_{r} v_{s}\right| \Psi_{\mathrm{in}}^{J J_{z}}\right\rangle= & \hat{S_{L_{A}}} \hat{J}_{A} \hat{J_{B}}\left\{\begin{array}{ccc}
S_{A} & S_{B} & S \\
L_{A} & 0 & L_{A} \\
J_{A} & S_{B} & J
\end{array}\right\}\left(S S_{z} L_{A}\left(J_{z}-S_{z}\right) \mid J J_{z}\right) \\
& \times\left\langle\Phi_{C} \Phi_{D}\left|v_{r}\right|\left(\Phi_{A} \Phi_{B}\right)_{\left(J_{z}-S_{z}\right)}^{L_{A}}\right\rangle\left\langle\left(\chi_{C} \chi_{D}\right)_{S_{z}}^{S}\left|v_{s}\right|\left(\chi_{A} \chi_{B}\right)_{S_{z}}^{S}\right\rangle,
\end{aligned}
$$

where $\left|S_{A}-S_{B}\right| \leq S \leq\left(S_{A}+S_{B}\right)$ and $\left|S-L_{A}\right| \leq J \leq\left(S+L_{A}\right)$. For the collision of unpolarized mesons, we can calculate the square of the matrix element, $\left|I_{\text {space-spin }}\right|^{2}$, average it over the initial states, and sum it over the final states. The result is

$$
\begin{aligned}
& \overline{\left|I_{\text {space-spin }}\right|^{2}}=\frac{1}{\left(2 J_{A}+1\right)\left(2 S_{B}+1\right)} \sum_{J J_{z} S S_{z}}\left(\hat{S} \hat{L}_{A} \hat{J}_{A} \hat{S_{B}}\right)^{2}\left\{\begin{array}{ccc}
S_{A} & S_{B} & S \\
L_{A} & 0 & L_{A} \\
J_{A} & S_{B} & J
\end{array}\right\}^{2} \\
& \times\left|\left(S S_{z} L_{A}\left(J_{z}-S_{z}\right) \mid J J_{z}\right)\right|^{2}\left|\sum_{i}^{3}\left\langle\Phi_{C} \Phi_{D}\left|v_{r}^{(i)}\right|\left(\Phi_{A} \Phi_{B}\right)_{\left(J_{z}-S_{z}\right)}^{L_{A}}\right\rangle\left\langle\left(\chi_{C} \chi_{D}\right)_{S_{z}}^{S}\left|v_{s}^{(i)}\right|\left(\chi_{A} \chi_{B}\right)_{S_{z}}^{S}\right\rangle\right|^{2} .
\end{aligned}
$$

The summation over $S_{z}$ can be carried out and the summation over $J_{z}$ can be converted to a summation over $M_{A}$. We then obtain

$$
\begin{aligned}
& \overline{\left|I_{\text {space-spin }}\right|^{2}=\sum_{S J M_{A}}(\hat{S} \hat{J})^{2}}\left\{\begin{array}{ccc}
S_{A} & S_{B} & S \\
L_{A} & 0 & L_{A} \\
J_{A} & S_{B} & J
\end{array}\right\}^{2} \\
& \times\left|\sum_{i}^{3}\left\langle\Phi_{C} \Phi_{D}\left|v_{r}^{(i)}\right|\left(\Phi_{A} \Phi_{B}\right)_{M_{A}}^{L_{A}}\right\rangle\left\langle\left(\chi_{C} \chi_{D}\right)_{S_{z}}^{S}\left|v_{s}^{(i)}\right|\left(\chi_{A} \chi_{B}\right)_{S_{z}}^{S}\right\rangle\right|^{2},
\end{aligned}
$$

where $-L_{A} \leq M_{A} \leq L_{A}$. From the relation between the matrix element of $V_{i j}\left(r_{i j}\right)$ and the cross section, the above result leads to the following "unpolarized" cross section for the collision of unpolarized mesons,

$$
\sigma^{\text {unpol }}=\sum_{S J M_{A}}(\hat{S} \hat{J})^{2}\left\{\begin{array}{ccc}
S_{A} & S_{B} & S \\
L_{A} & 0 & L_{A} \\
J_{A} & S_{B} & J
\end{array}\right\}^{2} \sigma\left(L_{A} M_{A} S S_{z}\right)
$$

where $\sigma\left(L_{A} M_{A} S S_{z}\right)$ is the cross section for the initial meson system to have a total internal orbital angular momentum $L_{A}$ and total spin $S$ with azimuthal components $M_{A}$ and $S_{z}$ respectively. For our interaction of Eq. (2), $\sigma\left(L_{A} M_{A} S S_{z}\right)$ is independent of $S_{z}$, and thus the label $S_{z}$ can be omitted. We can write out the results for other simple unpolarized cases. If $L_{A} \neq 0$ and $S_{B}=0$, then $S=S_{A}$ and the result of Eq. (15) becomes

$$
\sigma^{\text {unpol }}=\frac{1}{\left(2 L_{A}+1\right)} \sum_{M_{A}=-L_{A}}^{L_{A}} \sigma\left(L_{A} M_{A} S_{A}\right) .
$$

If $L_{A}=0$ and $S_{B} \neq 0$, then the result of Eq. (15) is

$$
\sigma^{\text {unpol }}=\frac{1}{\left(2 S_{A}+1\right)\left(2 S_{B}+1\right)} \sum_{S}(2 S+1) \sigma(S),
$$

where $\sigma(S)$ is the cross section when the initial two-meson system has a total spin $S$. 


\section{EVALUATION OF THE SPIN MATRIX ELEMENT}

We denote the spins of the constituents in the scattering process $A(12) B(34) \rightarrow$ $C(14) D(23)$ by $s_{1}, s_{2}, s_{3}$, and $s_{4}$. Using properties of the Wigner $\{9 j\}$ symbols [40, we may rearrange the spins to obtain

$$
\begin{aligned}
& \left|\left(\chi_{A} \chi_{B}\right)_{S_{z}}^{S}\right\rangle=\left|\left[\left(s_{1} s_{2}\right) S_{A}\left(s_{3} s_{4}\right) S_{B}\right]_{S_{z}}^{S}\right\rangle \\
= & (-1)^{S_{B}-s_{4}-s_{3}} \sum_{S_{14} S_{23}} \hat{S}_{A} \hat{S}_{B} \hat{S}_{14} \hat{S}_{23}\left\{\begin{array}{ccc}
s_{1} & s_{2} & S_{A} \\
s_{4} & s_{3} & S_{B} \\
S_{14} & S_{23} & S
\end{array}\right\}\left|\left[\left(s_{1} s_{4}\right) S_{14}\left(s_{2} s_{3}\right) S_{23}\right]_{S_{z}}^{S}\right\rangle
\end{aligned}
$$

The phase factor $(-1)^{S_{B}-s_{4}-s_{3}}$ arises from an interchange of spins in the Clebsch-Gordon coefficients,

$$
\left|\left(s_{3} s_{4}\right) S_{B}\right\rangle=(-1)^{S_{B}-s_{4}-s_{3}}\left|\left(s_{4} s_{3}\right) S_{B}\right\rangle
$$

The matrix element of the spin unit operator $v_{s}=1$ is then given by

$$
\begin{aligned}
& \left\langle\left(\chi_{C} \chi_{D}\right)_{S_{z}^{\prime}}^{S^{\prime}}\left|v_{s}\right|\left(\chi_{A} \chi_{B}\right)_{S_{z}}^{S}\right\rangle=\left\langle\left[\left(s_{1} s_{4}\right) S_{C}\left(s_{3} s_{2}\right) S_{D}\right]_{S_{z}^{\prime}}^{S^{\prime}}|1|\left[\left(s_{1} s_{2}\right) S_{A}\left(s_{3} s_{4}\right) S_{B}\right]_{S_{z}}^{S}\right\rangle \\
& =\delta_{S S^{\prime}} \delta_{S_{z} S_{z}^{\prime}}(-1)^{S_{B}+S_{D}-s_{2}-s_{4}-2 s_{3}} \hat{S}_{A} \hat{S}_{B} \hat{S}_{C} \hat{S}_{D}\left\{\begin{array}{ccc}
s_{1} & s_{2} & S_{A} \\
s_{4} & s_{3} & S_{B} \\
S_{C} & S_{D} & S
\end{array}\right\} .
\end{aligned}
$$

The matrix element of the operator $v_{s}=\mathbf{s}_{i} \cdot \mathbf{s}_{j}$ can be derived similarly. For diagrams C1 and $\mathrm{C} 2$, the matrix element is given by

$$
\begin{aligned}
& \left\langle\left(\chi_{C} \chi_{D}\right)_{S_{z}^{\prime}}^{S^{\prime}}\left|v_{s}\right|\left(\chi_{A} \chi_{B}\right)_{S_{z}}^{S}\right\rangle=\left\langle\left[\left(s_{1} s_{4}\right) S_{C}\left(s_{3} s_{2}\right) S_{D}\right]_{S_{z}^{\prime}}^{S^{\prime}}\left|\mathbf{s}_{i} \cdot \mathbf{s}_{j}\right|\left[\left(s_{1} s_{2}\right) S_{A}\left(s_{3} s_{4}\right) S_{B}\right]_{S_{z}}^{S}\right\rangle \\
& =\delta_{S S^{\prime}} \delta_{S_{z} S_{z}^{\prime}}(-1)^{S_{B}+S_{D}-s_{2}-s_{4}-2 s_{3}} \hat{S}_{A} \hat{S}_{B} \hat{S}_{C} \hat{S}_{D} \\
& \times\left\{\begin{array}{ccc}
s_{1} & s_{2} & S_{A} \\
s_{4} & s_{3} & S_{B} \\
S_{C} & S_{D} & S
\end{array}\right\} \frac{1}{2}\left[S_{i j}\left(S_{i j}+1\right)-S_{i}\left(S_{i}+1\right)-S_{j}\left(S_{j}+1\right)\right] .
\end{aligned}
$$

The values of $i, j$, and $S_{i j}$ for diagrams $\mathrm{C} 1$ and $\mathrm{C} 2$ are listed in Table I.

Table I. The values of $i, j$, and $S_{i j}$ in Eq. (21).

\begin{tabular}{|c|c|c|c|}
\hline Diagram & $i$ & $j$ & $S_{i j}$ \\
\hline Prior C1 & 1 & 4 & $S_{C}$ \\
Prior C2 & 2 & 3 & $S_{D}$ \\
\hline Post C1 & 1 & 2 & $S_{A}$ \\
Post C2 & 4 & 3 & $S_{B}$ \\
\hline
\end{tabular}

The matrix element of $v_{s}=\mathbf{s}_{i} \cdot \mathbf{s}_{j}$ for diagrams T1 and T2 is somewhat more complicated, and can be shown to be 


$$
\begin{aligned}
& \left.\left.\left\langle\left(\chi_{C} \chi_{D}\right)_{S_{z}^{\prime}}^{S^{\prime}}\left|v_{s}\right|\left(\chi_{A} \chi_{B}\right)_{S_{z}}^{S}\right\rangle=\left\langle\left[\left(s_{1} s_{4}\right) S_{C}\left(s_{3} s_{2}\right) S_{D}\right]\right]_{S_{z}}^{S}\left|\mathbf{s}_{i} \cdot \mathbf{s}_{j}\right|\left[\left(s_{1} s_{2}\right) S_{A}\left(s_{3} s_{4}\right) S_{B}\right]\right]_{S_{z}^{\prime}}^{S_{z}^{\prime}}\right\rangle \\
& =\delta_{S S^{\prime}} \delta_{S_{z} S_{z}^{\prime}} \sum_{S_{13}}(-1)^{S_{24}}{ }^{S_{24}-s_{4}-s_{2}}\left(2 S_{13}+1\right)\left(2 S_{24}+1\right) \hat{S}_{A} \hat{S}_{B} \hat{S}_{C} \hat{S}_{D} \\
& \times\left\{\begin{array}{cccc}
s_{1} & s_{2} & S_{A} \\
s_{3} & s_{4} & S_{B} \\
S_{13} & S_{24} & S
\end{array}\right\}\left\{\begin{array}{ccc}
s_{1} & s_{4} & S_{C} \\
s_{3} & s_{2} & S_{D} \\
S_{13} & S_{24} & S
\end{array}\right\} \frac{1}{2}\left[S_{i j}\left(S_{i j}+1\right)-S_{i}\left(S_{i}+1\right)-S_{j}\left(S_{j}+1\right)\right],
\end{aligned}
$$

where $i=1$ and $j=3$ for diagram T1, and $i=2$ and $j=4$ for diagram T2. The quantities $S_{13}$ and $S_{24}$ span the full allowed range in this summation.

Eqs. (20), (21), and (22) give the general results for the spin matrix element $I_{\text {spin }}$ of the unit operator and the $\mathbf{s}_{i} \cdot \mathbf{s}_{j}$ operator in a rearrangement collision. Our results agree with the explicit coefficients given in Table I of Barnes and Swanson [33].

\section{EVALUATION OF THE SPATIAL MATRIX ELEMENT}

In the quark-interchange reaction of Eq. (1), the masses of the quarks and antiquarks are different in general. Previously, meson scattering calculations with unequal masses using this approach had been discussed in detail in coordinate space [34]. Here we give the corresponding momentum space results for general quark and antiquark masses.

The spatial matrix element in Eq. (11) is

$$
\begin{aligned}
& \left\langle\left(\Phi_{C} \Phi_{D}\right)_{\left(J_{z}^{\prime}-S_{z}^{\prime}\right)}^{L^{\prime}}\left|v_{r}\right|\left(\Phi_{A} \Phi_{B}\right)_{\left(J_{z}-S_{z}\right)}^{L}\right\rangle \\
& =\sum_{M_{A} M_{B} M_{C} M_{D}}\left(L_{C} M_{C} L_{D} M_{D} \mid L^{\prime}\left(J_{z}^{\prime}-S_{z}^{\prime}\right)\right)^{*}\left(L_{A} M_{A} L_{B} M_{B} \mid L\left(J_{z}-S_{z}\right)\right) \\
& \left.\quad \times\left\langle\Phi_{C}\left(L_{C} M_{C}\right) \Phi_{D}\left(L_{D} M_{D}\right)\left|v_{r}\right| \Phi_{A}\left(L_{A} M_{A}\right) \Phi_{B}\left(L_{B} M_{B}\right)\right)\right\rangle .
\end{aligned}
$$

For the four diagrams in the reaction $A+B \rightarrow C+D$, the spatial matrix element

$$
I_{\text {space }}=\left\langle\Phi_{C}\left(L_{C} M_{C}\right) \Phi_{D}\left(L_{D} M_{D}\right)\left|v_{r}\right| \Phi_{A}\left(L_{A} M_{A}\right) \Phi_{B}\left(L_{B} M_{B}\right)\right)
$$

can be written in the form

$$
\begin{aligned}
I_{\text {space }}= & \iint d \boldsymbol{\kappa} d \boldsymbol{\kappa}^{\prime} \Phi_{A}\left[\zeta\left(2 \boldsymbol{k}_{A}-\boldsymbol{K}_{A}\right)\right] \Phi_{B}\left[\zeta\left(2 \boldsymbol{k}_{B}-\boldsymbol{K}_{B}\right)\right] \\
& \times \Phi_{C}\left[\zeta\left(2 \boldsymbol{k}_{C}-\boldsymbol{K}_{C}\right)\right] \Phi_{D}\left[\zeta\left(2 \boldsymbol{k}_{D}-\boldsymbol{K}_{D}\right)\right] V\left(\boldsymbol{\kappa}^{\prime}-\boldsymbol{\kappa}\right) .
\end{aligned}
$$

Here the momentum arguments are shown explicitly, and the angular momentum quantum numbers $L_{i}$ and $M_{i}$ for each meson are implicit. The quantity $\zeta= \pm 1$ is an overall sign which depends on the diagram (see Table II). The quantity $V(\boldsymbol{q})$, where $\boldsymbol{q}=\boldsymbol{\kappa}-\boldsymbol{\kappa}^{\prime}$, is the Fourier transform of the interaction $V_{i j}\left(r_{i j}\right)$ [the spin and spatial part of $H_{i j}\left(\boldsymbol{r}_{i j}\right)$ in Eq. (3)],

$$
V(\boldsymbol{q})=\int d \boldsymbol{r} e^{-i \boldsymbol{q} \cdot \boldsymbol{r}_{i j}} V_{i j}\left(r_{i j}\right) .
$$

The momenta $\boldsymbol{\kappa}$ is the initial three-momenta of the scattered constituent that is initially in meson $A$ and $\boldsymbol{\kappa}^{\prime}$ is its final three-momenta. The variables $\left\{\boldsymbol{k}_{i},(i=A, B, C, D)\right\}$ are either $\boldsymbol{\kappa}$ or $\boldsymbol{\kappa}^{\prime}$ depending on the diagram, as specified in Table II. We shall use the bold-faced 
symbols $\boldsymbol{A}, \boldsymbol{B}, \boldsymbol{C}$, and $\boldsymbol{D}$ to represent the momenta of $A, B, C$, and $D$ respectively. For simplicity we shall treat the scattering problem in the center-of-mass frame, so that $\boldsymbol{B}=-\boldsymbol{A}$ and $\boldsymbol{D}=-\boldsymbol{C}$. The quantity $\left\{\boldsymbol{K}_{i}\right\}$ is a function of $\boldsymbol{A}, \boldsymbol{C}$, and the mass parameter $f_{i}$, which is a function of the quark and antiquark masses in meson $i$. The function $\boldsymbol{K}_{i}\left(\boldsymbol{A}, \boldsymbol{C}, f_{i}\right)$ is tabulated for each diagram in Table II. For diagrams T1 and T2, the post and prior variables are identical and so do not need to be tabulated separately.

Table II. Diagram-dependent momentum arguments in post and prior formalisms.

\begin{tabular}{|c|c|c|c|c|c|c|c|c|c|}
\hline Diagram & $\zeta$ & $\boldsymbol{k}_{A}$ & $\boldsymbol{K}_{A}$ & $\boldsymbol{k}_{B}$ & $\boldsymbol{K}_{B}$ & $\boldsymbol{k}_{C}$ & $\boldsymbol{K}_{C}$ & $\boldsymbol{k}_{D}$ & $\boldsymbol{K}_{D}$ \\
\hline Prior C1 & 1 & $\boldsymbol{\kappa}$ & $f_{A} \boldsymbol{A}$ & $\boldsymbol{\kappa}$ & $f_{B} \boldsymbol{A}+2 \boldsymbol{C}$ & $\boldsymbol{\kappa}^{\prime}$ & $f_{C} \boldsymbol{C}$ & $\boldsymbol{\kappa}$ & $f_{D} \boldsymbol{C}+2 \boldsymbol{A}$ \\
Prior C2 & -1 & $\boldsymbol{\kappa}$ & $f_{A} \boldsymbol{A}$ & $\boldsymbol{\kappa}$ & $f_{B} \boldsymbol{A}-2 \boldsymbol{C}$ & $\boldsymbol{\kappa}$ & $-f_{C} \boldsymbol{C}+2 \boldsymbol{A}$ & $\boldsymbol{\kappa}^{\prime}$ & $-f_{D} \boldsymbol{C}$ \\
\hline Post C1 & 1 & $\boldsymbol{\kappa}$ & $f_{A} \boldsymbol{A}$ & $\boldsymbol{\kappa}^{\prime}$ & $f_{B} \boldsymbol{A}+2 \boldsymbol{C}$ & $\boldsymbol{\kappa}^{\prime}$ & $f_{C} \boldsymbol{C}$ & $\boldsymbol{\kappa}^{\prime}$ & $f_{D} \boldsymbol{C}+2 \boldsymbol{A}$ \\
Post C2 & -1 & $\boldsymbol{\kappa}$ & $f_{A} \boldsymbol{A}$ & $\boldsymbol{\kappa}^{\prime}$ & $f_{B} \boldsymbol{A}-2 \boldsymbol{C}$ & $\boldsymbol{\kappa}$ & $-f_{C} \boldsymbol{C}+2 \boldsymbol{A}$ & $\boldsymbol{\kappa}$ & $-f_{D} \boldsymbol{C}$ \\
\hline T1 & 1 & $\boldsymbol{\kappa}$ & $f_{A} \boldsymbol{A}$ & $\boldsymbol{\kappa}^{\prime}$ & $f_{B} \boldsymbol{A}+2 \boldsymbol{C}$ & $\boldsymbol{\kappa}^{\prime}$ & $f_{C} \boldsymbol{C}$ & $\boldsymbol{\kappa}$ & $f_{D} \boldsymbol{C}+2 \boldsymbol{A}$ \\
\hline T2 & -1 & $\boldsymbol{\kappa}$ & $f_{A} \boldsymbol{A}$ & $\boldsymbol{\kappa}^{\prime}$ & $f_{B} \boldsymbol{A}-2 \boldsymbol{C}$ & $\boldsymbol{\kappa}$ & $-f_{C} \boldsymbol{C}+2 \boldsymbol{A}$ & $\boldsymbol{\kappa}^{\prime}$ & $-f_{D} \boldsymbol{C}$ \\
\hline
\end{tabular}

The mass parameter $f_{i}$ is unity for mesons with equal quark and antiquark masses. For unequal masses, the $\left\{f_{i}\right\}$ are tabulated in Table III in terms of

$$
\Delta_{i}=\frac{m(q)_{i}-m(\bar{q})_{i}}{m(q)_{i}+m(\bar{q})_{i}}
$$

The $\left\{f_{i}\right\}$ are the same in post and prior formalisms.

Table III. The mass parameters $f_{i}$ for each diagram.

\begin{tabular}{|c|c|c|c|c|}
\hline Diagram= & C1 & C2 & T1 & T2 \\
\hline$f_{A}=$ & $1+\Delta_{A}$ & $1-\Delta_{A}$ & $1+\Delta_{A}$ & $1-\Delta_{A}$ \\
\hline$f_{B}=$ & $1-\Delta_{B}$ & $1+\Delta_{B}$ & $1-\Delta_{B}$ & $1+\Delta_{B}$ \\
\hline$f_{C}=$ & $1+\Delta_{C}$ & $1+\Delta_{C}$ & $1+\Delta_{C}$ & $1+\Delta_{C}$ \\
\hline$f_{D}=$ & $1-\Delta_{D}$ & $1-\Delta_{D}$ & $1-\Delta_{D}$ & $1-\Delta_{D}$ \\
\hline
\end{tabular}

To evaluate the spatial overlap integrals, we expand each meson wavefunction $\Phi(2 \mathbf{p})$ as a linear combination of (nonorthogonal) Gaussian basis functions $\phi_{n}(2 \boldsymbol{p})$ of different widths as

$$
\Phi(2 \boldsymbol{p})=\sum_{n=1}^{N} a_{n} \phi_{n}(2 \boldsymbol{p})
$$

where

$$
\phi_{n}(2 \boldsymbol{p})=N_{n}(2 p)^{l} \sqrt{\frac{4 \pi}{(2 l+1) ! !}} Y_{l m}(\hat{\boldsymbol{p}}) \exp \left\{-\frac{(2 \boldsymbol{p})^{2}}{8 n \beta^{2}}\right\} .
$$

This expansion makes the spatial integrals tractable. We choose to normalize the basis function $\phi_{n}(2 \boldsymbol{p})$ according to 


$$
\int d \boldsymbol{p}\left|\phi_{n}(\mathbf{2} \boldsymbol{p})\right|^{2}=1
$$

which leads to the normalization constant

$$
N_{n}=\left(\frac{1}{\pi n \beta^{2}}\right)^{3 / 4} \frac{1}{\left(2 n \beta^{2}\right)^{l / 2}} .
$$

We also normalize the meson wavefunction $\Phi(2 \boldsymbol{p})$ according to

$$
\int d \boldsymbol{p}|\Phi(\mathbf{2 p})|^{2}=1
$$

which implies a constraint on the coefficients $\left\{a_{n}\right\}$.

We shall first present our results for the spatial matrix element Eq. (25) for the case of all $S$-wave mesons, each with a single Gaussian wavefunction of the type of Eq. (29),

$$
\phi_{i}\left[\zeta\left(2 \boldsymbol{k}_{i}-\boldsymbol{K}_{i}\right)\right]=N_{i} \exp \left\{-\frac{\lambda_{i}}{2}\left(2 \boldsymbol{k}_{i}-\boldsymbol{K}_{i}\right)^{2}\right\}
$$

where $\lambda_{i}=1 / 4 n \beta^{2}$ and

$$
N_{i}=\sqrt{8}\left(\frac{\lambda_{i}}{\pi}\right)^{3 / 4}
$$

The product of wavefunctions in Eq. (25) is explicitly

$$
\begin{aligned}
& \phi_{A}\left[\zeta\left(2 \boldsymbol{k}_{A}-\boldsymbol{K}_{A}\right)\right] \phi_{B}\left[\zeta\left(2 \boldsymbol{k}_{B}-\boldsymbol{K}_{B}\right)\right] \phi_{C}\left[\zeta\left(2 \boldsymbol{k}_{C}-\boldsymbol{K}_{C}\right)\right] \phi_{D}\left[\zeta\left(2 \boldsymbol{k}_{D}-\boldsymbol{K}_{D}\right)\right] \\
& =N_{A} N_{B} N_{C} N_{D} \exp \left\{-\sum_{i=1}^{4} \frac{\lambda_{i}}{2}\left(2 \boldsymbol{k}_{i}-\boldsymbol{K}_{i}\right)^{2}\right\}
\end{aligned}
$$

The argument of the exponential, from the product of the four meson wavefunctions, is a function of $\boldsymbol{k}_{i}=\left\{\boldsymbol{\kappa}, \boldsymbol{\kappa}^{\prime}\right\}$ and the quantities $\left\{\boldsymbol{K}_{i}\right\}$. It can also be written as a function of $\boldsymbol{p}=\left(\boldsymbol{\kappa}+\boldsymbol{\kappa}^{\prime}\right) / 2$ and $\boldsymbol{q}=\boldsymbol{\kappa}^{\prime}-\boldsymbol{\kappa}$. In terms of $\boldsymbol{p}$ and $\boldsymbol{q}$, the $\left\{\boldsymbol{k}_{i}\right\}$ are given by

$$
\boldsymbol{k}_{i}=\boldsymbol{p}-\eta_{i} \boldsymbol{q} / 2
$$

where $\eta_{i}$ is

$$
\eta_{i}= \begin{cases}+1, & \text { if } \quad \boldsymbol{k}_{i}=\boldsymbol{\kappa} \\ -1, & \text { if } \quad \boldsymbol{k}_{i}=\boldsymbol{\kappa}^{\prime}\end{cases}
$$

Using Eq. (36) and completing the square in the exponential, we obtain

$$
\sum_{i=1}^{4} \frac{\lambda_{i}}{2}\left(2 \boldsymbol{k}_{i}-\boldsymbol{K}_{i}\right)^{2}=2 \sum_{i}^{4} \lambda_{i}\left(\boldsymbol{p}-\boldsymbol{p}_{0}\right)^{2}+\frac{\mu}{2}\left(\boldsymbol{q}-\boldsymbol{q}_{0}\right)^{2}+\frac{\nu}{2}
$$

where the quantities $\boldsymbol{p}_{0}, \boldsymbol{q}_{0}, \mu$, and $\nu$ are defined below. The shift $\boldsymbol{p}_{0}$ is

$$
\boldsymbol{p}_{0}=r_{0} \boldsymbol{q}+\mathbf{s}_{0}
$$


where $r_{0}$ and $\mathbf{s}_{0}$ are

$$
\begin{gathered}
r_{0}=\sum_{i=1}^{4} \eta_{i} \lambda_{i} / 2 \sum_{i=1}^{4} \lambda_{i}, \\
\mathbf{s}_{0}=\sum_{i=1}^{4} \eta_{i} \boldsymbol{K}_{i} / 2 \sum_{i=1}^{4} \lambda_{i} .
\end{gathered}
$$

The quantity $\mu$ in Eq. (38) is

$$
\mu=4\left(\sum_{i=1}^{4} \frac{1+\eta_{i}}{2} \lambda_{i}\right)\left(\sum_{j=1}^{4} \frac{1-\eta_{j}}{2} \lambda_{j} / \sum_{i=1}^{4} \lambda_{i}\right)
$$

the shift $\boldsymbol{q}_{0}$ is

$\boldsymbol{q}_{0}=-\frac{2}{\mu \sum_{i=1}^{4} \lambda_{i}}\left[\left(\sum_{i=1}^{4} \frac{1-\eta_{i}}{2} \lambda_{i}\right)\left(\sum_{j=1}^{4} \frac{1+\eta_{j}}{2} \lambda_{j} \boldsymbol{K}_{j}\right)-\left(\sum_{i=1}^{4} \frac{1+\eta_{i}}{2} \lambda_{i}\right)\left(\sum_{j=1}^{4} \frac{1-\eta_{j}}{2} \lambda_{j} \boldsymbol{K}_{j}\right)\right]$,

and $\nu$ is

$$
\nu=\sum_{i=1}^{4} \lambda_{i} \boldsymbol{K}_{i}^{2}-4 \sum_{i=1}^{4} \lambda_{i} \mathbf{s}_{0}^{2}-\mu \boldsymbol{q}_{0}^{2}
$$

The product of wavefunctions in Eq. (25) can therefore be written in a shifted Gaussian form,

$$
\begin{gathered}
\phi_{A}\left[\zeta\left(2 \boldsymbol{k}_{A}-\boldsymbol{K}_{A}\right)\right] \phi_{B}\left[\zeta\left(2 \boldsymbol{k}_{B}-\boldsymbol{K}_{B}\right)\right] \phi_{C}\left[\zeta\left(2 \boldsymbol{k}_{C}-\boldsymbol{K}_{C}\right)\right] \phi_{D}\left[\zeta\left(2 \boldsymbol{k}_{D}-\boldsymbol{K}_{D}\right)\right] \\
=N_{A} N_{B} N_{C} N_{D} \exp \left\{-2 \sum_{i=1}^{4} \lambda_{i}\left(\boldsymbol{p}-\boldsymbol{p}_{0}\right)^{2}-\frac{\mu}{2}\left(\boldsymbol{q}-\boldsymbol{q}_{0}\right)^{2}-\frac{\nu}{2}\right\} .
\end{gathered}
$$

The spatial matrix element of Eq. (25) then becomes

$$
\begin{array}{r}
\iint d \boldsymbol{\kappa} d \boldsymbol{\kappa}^{\prime} \phi_{A}\left[\zeta\left(2 \boldsymbol{k}_{A}-\boldsymbol{K}_{A}\right)\right] \phi_{B}\left[\zeta\left(2 \boldsymbol{k}_{B}-\boldsymbol{K}_{B}\right)\right] \phi_{C}\left[\zeta\left(2 \boldsymbol{k}_{C}-\boldsymbol{K}_{C}\right)\right] \phi_{D}\left[\zeta\left(2 \boldsymbol{k}_{D}-\boldsymbol{K}_{D}\right)\right] V(\boldsymbol{q}) \\
=\iint d \boldsymbol{p} d \boldsymbol{q} N_{A} N_{B} N_{C} N_{D} \exp \left\{-2 \sum_{i=1}^{4} \lambda_{i}\left(\boldsymbol{p}-\boldsymbol{p}_{0}\right)^{2}-\frac{\mu}{2}\left(\boldsymbol{q}-\boldsymbol{q}_{0}\right)^{2}-\frac{\nu}{2}\right\} V(\boldsymbol{q}) .
\end{array}
$$

The integration over $\boldsymbol{p}$ can be carried out analytically, which gives

$$
\iint d \boldsymbol{p} d \boldsymbol{q} \prod_{i=1}^{4} \phi_{i}\left[\zeta\left(2 \boldsymbol{k}_{i}-\boldsymbol{K}_{i}\right)\right] V(\boldsymbol{q})=N_{A} N_{B} N_{C} N_{D}\left(\frac{\pi}{2 \sum_{i=1}^{4} \lambda_{i}^{4}}\right)^{3 / 2} e^{-\frac{\nu}{2}} \int d \boldsymbol{q} e^{-\frac{\mu}{2}\left(\boldsymbol{q}-\boldsymbol{q}_{0}\right)^{2}} V(\boldsymbol{q}) .
$$

Thus, the six-dimensional integral of Eq. (46) is simplified to a three-dimensional integral involving an integration over $V(\boldsymbol{q})$.

The interaction $V(\boldsymbol{q})$, which we take from the standard quark model $V_{i j}(r)$, is the sum of Fourier transforms of color-Coulomb, spin-spin contact, linear confinement and constant 
terms. The sum of all diagrams arising from the constant term is zero for the scattering problem, so we do not need to include $V_{\text {con }}$ in deriving scattering amplitudes.

For the remaining three interactions, we have (using some integrals of Ref. [38])

$$
\begin{aligned}
\int d \boldsymbol{q} e^{-\frac{\mu}{2}\left(\boldsymbol{q}-\boldsymbol{q}_{0}\right)^{2}} V_{\text {Cou. }}(\boldsymbol{q}) & =\int d \boldsymbol{q} e^{-\frac{\mu}{2}\left(\boldsymbol{q}-\boldsymbol{q}_{0}\right)^{2}} \frac{4 \pi \alpha_{s}}{\boldsymbol{q}^{2}} \\
& =\frac{4 \pi \alpha_{s}(2 \pi)^{3 / 2}}{\sqrt{\mu}} e^{-\mu \boldsymbol{q}_{0}^{2} / 2}{ }_{1} F_{1}\left(\frac{1}{2}, \frac{3}{2}, \frac{\mu \mathbf{q}_{0}^{2}}{2}\right), \\
\int d \boldsymbol{q} e^{-\frac{\mu}{2}\left(\boldsymbol{q}-\boldsymbol{q}_{0}\right)^{2}} V_{\mathrm{Ss}}(\mathbf{q}) & =\int d \boldsymbol{q} e^{-\frac{\mu}{2}\left(\boldsymbol{q}-\boldsymbol{q}_{0}\right)^{2}}\left(\frac{-8 \pi \alpha_{s}}{3 m_{i} m_{j}}\right) e^{-\boldsymbol{q}^{2} / 4 \sigma^{2}} \\
& =\frac{-8 \pi \alpha_{s}}{3 m_{i} m_{j}}\left(\frac{2 \pi}{\mu}\right)^{3 / 2}\left(\frac{2 \sigma^{2} \mu}{1+2 \sigma^{2} \mu}\right)^{3 / 2} \exp \left\{-\frac{\mu \boldsymbol{q}_{0}^{2}}{2\left(1+2 \sigma^{2} \mu\right)}\right\},
\end{aligned}
$$

and

$$
\int d \boldsymbol{q} e^{-\frac{\mu}{2}\left(\boldsymbol{q}-\boldsymbol{q}_{0}\right)^{2}} V_{\operatorname{lin}}(\mathbf{q})=\left(-\frac{3}{4}\right) 8 \pi b\left(\frac{2 \pi}{3}\right)^{3 / 2} \mu^{2} e^{-\mu \boldsymbol{q}_{o}^{2} / 2}{ }_{1} F_{1}\left(-\frac{1}{2}, \frac{3}{2}, \frac{\mu \boldsymbol{q}_{0}^{2}}{2}\right)
$$

where $V_{\text {lin }}(\boldsymbol{q})=(-3 / 4) \int d \mathbf{r} e^{-i \boldsymbol{q} \cdot \mathbf{r}} b r$. These results allow one to evaluate the transition matrix elements $T_{f i}$ explicitly for the different interactions in diagrams C1, C2, T1, and T2 for the case of Gaussian meson wavefunctions.

The wavefunctions we employ here are in general sums of Gaussians of different widths (Eq. (28)). Eq. (25) can be evaluated in that case as well, so that the spatial matrix element Eq. (25) becomes

$$
\begin{aligned}
& I_{\text {space }} \\
& =\iint d \boldsymbol{\kappa} d \boldsymbol{\kappa}^{\prime} \Phi_{A}\left[\zeta\left(2 \boldsymbol{k}_{A}-\boldsymbol{K}_{A}\right)\right] \Phi_{B}\left[\zeta\left(2 \boldsymbol{k}_{B}-\boldsymbol{K}_{B}\right)\right] \Phi_{C}\left[\zeta\left(2 \boldsymbol{k}_{C}-\boldsymbol{K}_{C}\right)\right] \Phi_{D}\left[\zeta\left(2 \boldsymbol{k}_{D}-\boldsymbol{K}_{D}\right)\right] V\left(\boldsymbol{\kappa}^{\prime}-\boldsymbol{\kappa}\right) \\
& =\sum_{n_{A}=1}^{N} \sum_{n_{B}=1}^{N} \sum_{n_{C}=1}^{N} \sum_{n_{D}=1}^{N} a_{n_{A}} a_{n_{B}} a_{n_{C}} a_{n_{D}} I_{\text {space }}\left(n_{A}, n_{B}, n_{C}, n_{D}\right)
\end{aligned}
$$

where

$$
\begin{aligned}
I_{\text {space }}\left(n_{A}, n_{B}, n_{C}, n_{D}\right)= & \iint d \boldsymbol{\kappa} d \boldsymbol{\kappa}^{\prime} \phi_{A n_{A}}\left[\zeta\left(2 \boldsymbol{k}_{A}-\boldsymbol{K}_{A}\right)\right] \phi_{B n_{B}}\left[\zeta\left(2 \boldsymbol{k}_{B}-\boldsymbol{K}_{B}\right)\right] \\
& \times \phi_{C n_{C}}\left[\zeta\left(2 \boldsymbol{k}_{C}-\boldsymbol{K}_{C}\right)\right] \phi_{D n_{D}}\left[\zeta\left(2 \boldsymbol{k}_{D}-\boldsymbol{K}_{D}\right)\right] V\left(\boldsymbol{\kappa}^{\prime}-\boldsymbol{\kappa}\right) .
\end{aligned}
$$

$I_{\text {space }}\left(n_{A}, n_{B}, n_{C}, n_{C}\right)$ is the previous result of Eq. (25) for a single component wavefunction. The overlap integral in the multicomponent case is simply a sum of single-component contributions, each weighed by a coefficient product $a_{n_{A}} a_{n_{B}} a_{n_{C}} a_{n_{D}}$.

After the matrix elements for the interaction (2) are evaluated, the cross section for the process $A+B \rightarrow C+D$ can then be obtained using conventional scattering theory, as discussed in Section II. 


\section{EVALUATION OF THE SPATIAL MATRIX ELEMENT FOR AN $L=1$ MESON}

In the last section, we considered the scattering of $S$-wave $(L=0)$ mesons. Here we generalize to collisions in which a $P$-wave $(L=1)$ meson $A$ collides with an $S$-wave meson $B$, and scatter into two $S$-wave mesons $C$ and $D$.

First we consider single-component Gaussian wavefunctions. (The results can be easily generalized to multicomponent Gaussian wavefunctions.) Eq. (25) becomes

$$
\begin{aligned}
& \iint d \boldsymbol{\kappa} d \boldsymbol{\kappa}^{\prime} \phi_{A}\left(2 \boldsymbol{k}_{A}-\boldsymbol{K}_{A}\right) \phi_{B}\left(2 \boldsymbol{k}_{B}-\boldsymbol{K}_{B}\right) \phi_{C}\left(2 \boldsymbol{k}_{C}-\boldsymbol{K}_{C}\right) \phi_{D}\left(2 \boldsymbol{k}_{D}-\boldsymbol{K}_{D}\right) V(\boldsymbol{q}) \\
& =\iint d \boldsymbol{\kappa} d \boldsymbol{\kappa}^{\prime} N_{A}\left|2 \boldsymbol{p}_{A}\right|^{L_{A}} \sqrt{\frac{4 \pi}{\left(2 L_{A}+1\right) ! !}} Y_{L_{A} M_{A}}\left(\hat{\boldsymbol{p}}_{A}\right) \exp \left\{-\frac{\lambda_{i}}{2}\left(2 \boldsymbol{k}_{A}-\boldsymbol{K}_{A}\right)^{2}\right\} \\
& \times \phi_{B}\left(2 \boldsymbol{k}_{B}-\boldsymbol{K}_{B}\right) \phi_{C}\left(2 \boldsymbol{k}_{C}-\boldsymbol{K}_{C}\right) \phi_{D}\left(2 \boldsymbol{k}_{D}-\boldsymbol{K}_{D}\right) V(\boldsymbol{q}),
\end{aligned}
$$

where $2 \boldsymbol{p}_{A}=2 \boldsymbol{k}_{A}-\boldsymbol{K}_{A}$. Setting $L_{A}=1$ for the $P$-wave meson $A$, we have

$$
\left|2 \boldsymbol{k}_{A}-\boldsymbol{K}_{A}\right|^{L_{A}} \sqrt{\frac{4 \pi}{\left(2 L_{A}+1\right) ! !}} Y_{L_{A} M_{A}}\left(\hat{\boldsymbol{p}}_{A}\right)=\left\{\begin{array}{ll}
2 \kappa_{z}-f_{A} A_{z} & \text { if } M_{A}=0 \\
-\left(2 \kappa_{x}-f_{A} A_{x}\right)-i\left(2 \kappa_{y}-f_{A} A_{y}\right) & \text { if } M_{A}=1 . \\
\left(2 \kappa_{x}-f_{A} A_{x}\right)-i\left(2 \kappa_{y}-f_{A} A_{y}\right) & \text { if } M_{A}=-1
\end{array} .\right.
$$

It then suffices to evaluate

$$
\begin{aligned}
& \iint d \boldsymbol{\kappa} d \boldsymbol{\kappa}^{\prime}\left(2 \boldsymbol{\kappa}-f_{A} \boldsymbol{A}\right)_{k} N_{A} \exp \left\{-\frac{\lambda_{A}}{2}\left(2 \boldsymbol{k}_{A}-\boldsymbol{K}_{A}\right)^{2}\right\} \\
& \times \phi_{B}\left(2 \boldsymbol{k}_{B}-\boldsymbol{K}_{B}\right) \phi_{C}\left(2 \boldsymbol{k}_{C}-\boldsymbol{K}_{C}\right) \phi_{D}\left(2 \boldsymbol{k}_{D}-\boldsymbol{K}_{D}\right) V(\boldsymbol{q}) \\
&=\iint d \boldsymbol{p} d \boldsymbol{q}\left(2 \boldsymbol{\kappa}-f_{A} \boldsymbol{A}\right)_{k} N_{A} N_{B} N_{C} N_{D} \exp \left\{-2 \sum_{i=1}^{4} \lambda_{i}\left(\boldsymbol{p}-\boldsymbol{p}_{0}\right)^{2}-\frac{\mu}{2}\left(\boldsymbol{q}-\boldsymbol{q}_{0}\right)^{2}-\frac{\nu}{2}\right\} V(\boldsymbol{q}) .
\end{aligned}
$$

We can express $2 \boldsymbol{\kappa}-f_{A} \boldsymbol{A}$ in terms of $\boldsymbol{p}$ and $\boldsymbol{q}$;

$$
2 \boldsymbol{\kappa}-f_{A} \boldsymbol{A}=2 \boldsymbol{p}-\boldsymbol{q}-f_{A} \boldsymbol{A}=2\left(\boldsymbol{p}-\boldsymbol{p}_{0}\right)+2 \boldsymbol{p}_{0}-\boldsymbol{q}-f_{A} \boldsymbol{A} .
$$

Substituting Eq. (39) into this result, we find

$$
2 \boldsymbol{\kappa}-f_{A} \boldsymbol{A}=2\left(\boldsymbol{p}-\boldsymbol{p}_{0}\right)+\left(2 r_{0}-1\right)\left(\boldsymbol{q}-\boldsymbol{q}_{0}\right)+\left(2 r_{0}-1\right) \boldsymbol{q}_{0}+2 \mathbf{s}_{0}-f_{A} \boldsymbol{A} .
$$

The integral of $\boldsymbol{p}-\boldsymbol{p}_{0}$ gives zero. The integration over the last three terms can be carried

out in the same way as in the $L_{A}=0$ case, because $\boldsymbol{q}_{0}, \mathbf{s}_{0}$ and $\boldsymbol{A}$ are independent of the integration variables. It is thus only necessary to evaluate the integral

$$
\iint d \boldsymbol{p} d \boldsymbol{q} N_{A} N_{B} N_{C} N_{D}\left(\boldsymbol{q}-\boldsymbol{q}_{0}\right)_{k} \exp \left\{-2 \sum_{i=1}^{4} \lambda_{i}\left(\boldsymbol{p}-\boldsymbol{p}_{0}\right)^{2}-\frac{\mu}{2}\left(\boldsymbol{q}-\boldsymbol{q}_{0}\right)^{2}-\frac{\nu}{2}\right\} V(\boldsymbol{q}) .
$$

The integration over $\boldsymbol{p}$ can be carried out analytically, which gives

$$
\begin{aligned}
& \iint d \boldsymbol{p} d \boldsymbol{q} N_{A} N_{B} N_{C} N_{D}\left(\boldsymbol{q}-\boldsymbol{q}_{0}\right)_{k} \exp \left\{-2 \sum_{i=1}^{4} \lambda_{i}\left(\boldsymbol{p}-\boldsymbol{p}_{0}\right)^{2}-\frac{\mu}{2}\left(\boldsymbol{q}-\boldsymbol{q}_{0}\right)^{2}-\frac{\nu}{2}\right\} V(\boldsymbol{q}) \\
= & N_{A} N_{B} N_{C} N_{D}\left(\frac{\pi}{2 \sum_{i=1}^{4} \lambda_{i}^{4}}\right)^{3 / 2} e^{-\frac{\nu}{2}} \frac{1}{\mu} \frac{\partial}{\partial q_{0 k}} \int d \boldsymbol{q} e^{-\frac{\mu}{2}\left(\boldsymbol{q}-\boldsymbol{q}_{0}\right)^{2}} V(\boldsymbol{q}) .
\end{aligned}
$$


The $d \boldsymbol{q}$ integrals for the various potentials have already been obtained in closed form, and the differentiation with respect to $q_{0 k}$ is straightforward. We then find

$$
\begin{aligned}
& \frac{\partial}{\partial q_{0 k}} \int d \boldsymbol{q} e^{-\frac{\mu}{2}\left(\boldsymbol{q}-\boldsymbol{q}_{0}\right)^{2}} V_{\text {Cou. }}(\boldsymbol{q}) \\
&=\frac{4 \pi \alpha_{s}(2 \pi)^{3 / 2}}{\sqrt{\mu}} e^{-\mu \boldsymbol{q}_{0}^{2} / 2}\left[-{ }_{1} F_{1}\left(\frac{1}{2}, \frac{3}{2}, \frac{\mu \boldsymbol{q}_{0}^{2}}{2}\right)+\frac{1}{3}{ }_{1} F_{1}\left(\frac{3}{2}, \frac{5}{2}, \frac{\mu \boldsymbol{q}_{0}^{2}}{2}\right)\right] \mu q_{0 k}, \\
& \frac{\partial}{\partial q_{0 k}} \int d \boldsymbol{q} e^{-\frac{\mu}{2}\left(\boldsymbol{q}-\boldsymbol{q}_{0}\right)^{2}} V_{\mathrm{ss}}(\mathbf{q})=\frac{-8 \pi \alpha_{s}}{3 m_{i} m_{j}}\left(\frac{2 \pi}{\mu}\right)^{3 / 2}\left(\frac{2 \sigma^{2} \mu}{1+2 \sigma^{2} \mu}\right)^{3 / 2} \exp \left\{-\frac{\mu \boldsymbol{q}_{0}^{2}}{2\left(1+2 \sigma^{2} \mu\right)}\right\} \\
& \times\left(-\frac{\mu q_{o i}}{1+2 \sigma^{2} \mu}\right),
\end{aligned}
$$

and

$$
\begin{aligned}
\frac{\partial}{\partial q_{0 k}} \int d \boldsymbol{q} e^{-\frac{\mu}{2}\left(\boldsymbol{q}-\boldsymbol{q}_{0}\right)^{2}} V_{\operatorname{lin}}(\mathbf{q})= & \left(-\frac{3}{4}\right) b 8 \pi\left(\frac{2 \pi}{3}\right)^{3 / 2} \mu^{2} e^{-\mu \boldsymbol{q}_{o}^{2} / 2} \\
& \times\left[-{ }_{1} F_{1}\left(-\frac{1}{2}, \frac{3}{2}, \frac{\mu \boldsymbol{q}_{0}^{2}}{2}\right)-\frac{1}{3}{ }_{1} F_{1}\left(\frac{1}{2}, \frac{5}{2}, \frac{\mu \boldsymbol{q}_{0}^{2}}{2}\right)\right] \mu q_{0 k} .
\end{aligned}
$$

The scattering amplitude $T_{f i}$ and cross section $\sigma_{f i}$ for the dissociation of a $P$-wave meson through an $S P \rightarrow S S$ reaction will subsequently be evaluated using these results.

\section{MESON WAVE FUNCTIONS}

In nonrelativistic reaction theory, the equality of the scattering amplitude for rearrangement reactions in post and prior formalisms follows if and only if the two-body interaction used in evaluating the scattering matrix elements is identical to the interaction that generates the bound state wavefunctions. If this is not the case, the post and prior scattering amplitudes will differ. It is therefore especially important to determine accurate bound state wavefunctions in evaluating scattering amplitudes. For this purpose, we will first search for a set of interaction Hamiltonian parameters that fit the known meson spectrum with reasonable accuracy. This interaction and the associated bound state wavefunctions will be used in our subsequent reaction calculations.

For a bound state of quark $i$ and antiquark $j$ of momentum $\boldsymbol{p}_{i}$ and $\boldsymbol{p}_{j}$ and reduced momentum $\boldsymbol{p}$,

$$
\boldsymbol{p}=\frac{m_{j} \boldsymbol{p}_{i}-m_{i} \boldsymbol{p}_{j}}{m_{i}+m_{j}}
$$

the Hamiltonian is

$$
H=\frac{\boldsymbol{p}^{2}}{2 \mu}+V(r)
$$

where $\mu$ is the reduced mass $m_{i} m_{j} /\left(m_{i}+m_{j}\right)$, and $V(r)$ is the quark-antiquark interaction, (see $H_{i j}$ of Eq. (2)), 


$$
V(r)=-\frac{\boldsymbol{\lambda}(i)}{2} \cdot \frac{\boldsymbol{\lambda}^{T}(j)}{2}\left\{\frac{\alpha_{s}}{r}-\frac{3 b}{4} r-\frac{8 \pi \alpha_{s}}{3 m_{i} m_{j}} \boldsymbol{s}_{i} \cdot \boldsymbol{s}_{j}\left(\frac{\sigma^{3}}{\pi^{3 / 2}}\right) e^{-\sigma^{2} r^{2}}+V_{\text {con }}\right\} .
$$

For a quark and antiquark in a color-singlet hadron, the matrix element of $-\boldsymbol{\lambda}(i) \cdot \boldsymbol{\lambda}^{T}(j) / 4$ is the familiar color factor $C_{f}=-4 / 3$.

For given orbital angular momentum quantum numbers $l$ and $m$, the eigenstate $\Phi(2 \boldsymbol{p})$ of this Hamiltonian can be represented as the expansion in Eq. (28) in the set of (nonorthogonal) Gaussian basis states $\left\{\phi_{n}\right\}$ of Eq. (29) with expansion coefficients $\left\{a_{n}\right\}$. The eigenvalue equation $H \Phi=E \Phi$ then becomes the matrix equation

$$
\mathcal{H} a=E B a
$$

where $a$ is a column matrix with elements $\left\{a_{1}, a_{2}, \ldots, a_{N}\right\}, B$ is the symmetric matrix

$$
B_{i j}=\langle i \mid j\rangle \equiv \int d \boldsymbol{p} \phi_{i}^{*}(2 \boldsymbol{p}) \phi_{j}(2 \boldsymbol{p})=\left(\frac{2 \sqrt{i j}}{i+j}\right)^{l+3 / 2}
$$

and $\mathcal{H}$ is the Hamiltonian matrix

$$
\mathcal{H}_{i j}=T_{i j}+V_{i j}
$$

which is the sum of the kinetic energy matrix $T_{i j}$

$$
T_{i j}=\int d \boldsymbol{p} \phi_{i}^{*}(2 \boldsymbol{p}) \frac{\boldsymbol{p}^{2}}{2 \mu} \phi_{j}(2 \boldsymbol{p})=(2 l+3) \frac{i j}{i+j}\left(\frac{2 \sqrt{i j}}{i+j}\right)^{l+3 / 2} \frac{\beta^{2}}{2 \mu},
$$

and the potential matrix $V_{i j}$

$$
V_{i j} \equiv\langle i|V| j\rangle=(2 \pi)^{3} \int d \boldsymbol{r} \tilde{\phi}_{i}^{*}(\boldsymbol{r}) V(\boldsymbol{r}) \tilde{\phi}_{j}(\boldsymbol{r}),
$$

where $\tilde{\phi}_{i}(\boldsymbol{r})$ is the Fourier transform of $\phi_{i}(2 \boldsymbol{p})$,

$$
\tilde{\phi}_{i}(\boldsymbol{r})=\int \frac{d \boldsymbol{p}}{(2 \pi)^{3}} e^{i \boldsymbol{p} \cdot \boldsymbol{r}} \phi_{i}(2 \boldsymbol{p}) \text {. }
$$

Evaluation of the potential matrix elements for the color-Coulomb interaction gives

$$
\begin{aligned}
V_{i j}(\text { Cou. }) & =C_{f} 4 \pi \alpha_{s}\left\langle i\left|\frac{1}{r}\right| j\right\rangle \\
& =C_{f} \frac{4 \pi \alpha_{s} \beta}{(2 \pi)^{3 / 2}} \frac{2^{l} l !}{(2 l+1) ! !} \sqrt{i+j}\left(\frac{2 \sqrt{i j}}{i+j}\right)^{l+3 / 2} .
\end{aligned}
$$

For the linear interaction we similarly find

$$
\begin{aligned}
V_{i j}(\text { lin. }) & =\left\langle i\left|C_{f}\left(-\frac{3}{4}\right) b r\right| j\right\rangle \\
& =C_{f}\left(-\frac{3}{4}\right) \frac{b}{\beta} \frac{8 \pi}{(2 \pi)^{3 / 2}} \frac{(l+1) !}{(2 l+1) ! !} \frac{1}{\sqrt{i+j}}\left(\frac{2 \sqrt{i j}}{i+j}\right)^{l+3 / 2}
\end{aligned}
$$


and for the spin-spin interaction we find

$$
\begin{aligned}
V_{i j}(\mathrm{ss}) & =-C_{f} \frac{8 \pi \alpha_{s}}{3 m_{i} m_{j}}\left(\frac{\sigma^{3}}{\pi^{3 / 2}}\right)\left\langle i\left|\boldsymbol{s}_{i} \cdot \boldsymbol{s}_{j} e^{-\sigma^{2} r^{2}}\right| j\right\rangle \\
& =-C_{f} \frac{8 \pi \alpha_{s}}{3 m_{i} m_{j}} \boldsymbol{s}_{i} \cdot \boldsymbol{s}_{j}\left(\frac{\sigma^{3}}{\pi^{3 / 2}}\right)\left(\frac{2 \sqrt{i j}}{i+j+2 \sigma^{2} / \beta^{2}}\right)^{l+3 / 2} .
\end{aligned}
$$

Given these Hamiltonian matrix elements, the eigenvalues and eigenvectors can be obtained from the eigenvalue equation (65). In our numerical calculations we used a six-component $(N=6)$ space of Gaussian basis functions.

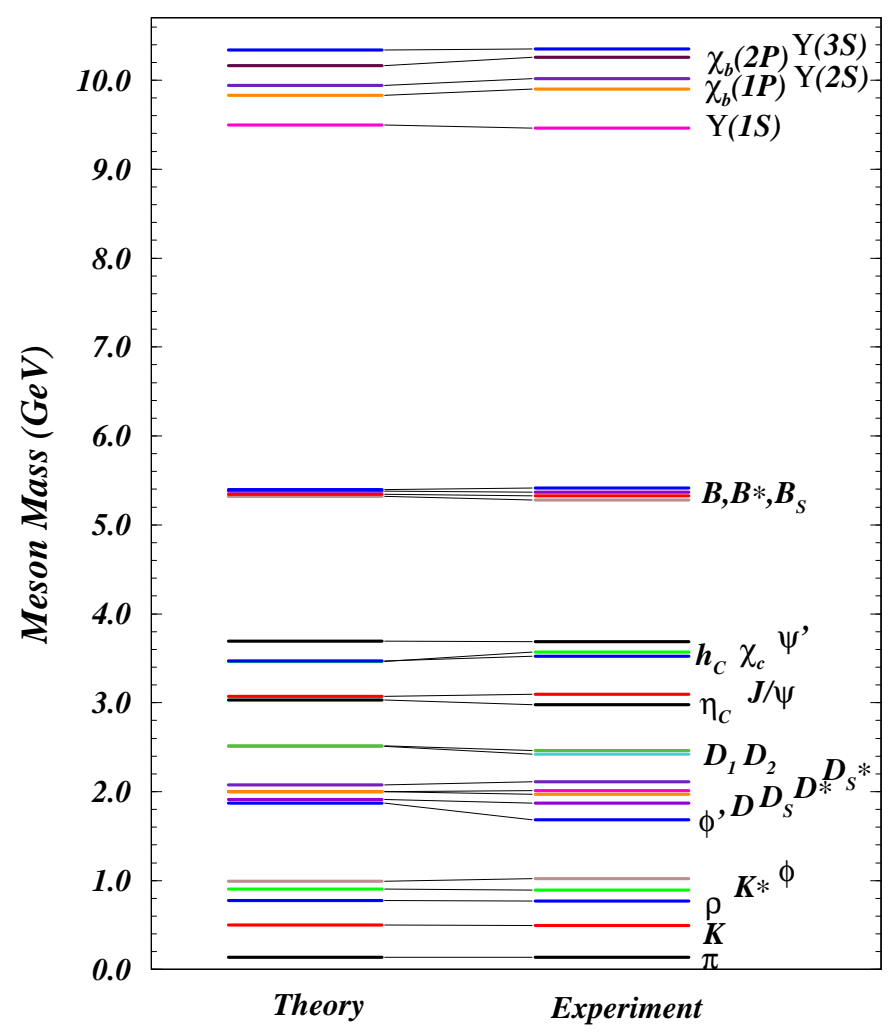

Fig. 3. Comparison of experimental energy levels and theoretical energy levels calculated with the parameter set of Eq. (74).

For this study we assumed a running coupling constant combined with an otherwise fairly conventional quark model parameter set, given by

$$
\begin{aligned}
\alpha_{s}\left(Q^{2}\right) & =\frac{12 \pi}{\left(33-2 n_{f}\right) \ln \left(A+Q^{2} / B^{2}\right)}, \quad A=10, \quad B=0.31 \mathrm{GeV}, \\
b & =0.18 \mathrm{GeV}^{2}, \quad \sigma=0.897 \mathrm{GeV}, \quad m_{u}=m_{d}=0.334 \mathrm{GeV}, \\
m_{s} & =0.575 \mathrm{GeV}, \quad m_{c}=1.776 \mathrm{GeV}, \quad m_{b}=5.102 \mathrm{GeV}, \\
V_{\text {con }} & =-0.620 \mathrm{GeV} .
\end{aligned}
$$

We identified the scale $Q^{2}$ in the running coupling constant with the square of the invariant mass of the interacting constituents, $Q^{2}=s_{i j}$. This set of parameters leads to a meson 
spectrum which is reasonably close to experiment (see Fig. 3); the theoretical masses and wavefunctions are given in Appendix A. The parameter set used earlier in 21 is similar to this set but it has a fixed strong coupling constant. An alternative set of quark model parameters, without a running coupling constant, was used for comparison. This second set was $\alpha_{s}=0.594, b=0.162 \mathrm{GeV}^{2}, \sigma=0.897 \mathrm{GeV}, m_{u}=m_{d}=0.335 \mathrm{GeV}$, and $m_{c}=$ $1.6 \mathrm{GeV}$, and a flavor-dependent $V_{\text {con }}$.

Having obtained the set of coefficients $\left\{a_{n}\right\}$ for each initial and final meson, we can proceed to the calculation of the scattering amplitudes $T_{f i}$ and the dissociation cross sections $\sigma_{f i}$. Some explanation of the evaluation of our (somewhat arbitrary) choice of momentum scale $Q^{2}=s_{i j}$ in the running coupling constant $\alpha\left(Q^{2}\right)$ in Eq. (74) for the scattering problem is appropriate. For a reaction process involving the interaction of constituent $i=a$ in meson $A\left(a a^{\prime}\right)$ with $j=b$ in meson $B\left(b b^{\prime}\right)$, we can determine the invariant mass squared $s_{i j}$ of $a$ and $b$ as follows: Constituent $a$ carries a fraction $x_{+}$of the forward light-cone momentum of the initial meson $A$, and $b$ carries a fraction $x_{-}$of the backward light-cone momentum of initial meson $B$. For simplicity, we assume that the momentum fraction carried by a constituent is proportional to its rest mass, which is exact in the weak binding limit;

$$
\begin{gathered}
x_{+}=\frac{m_{a}}{m_{a}+m_{a^{\prime}}}, \\
x_{-}=\frac{m_{b}}{m_{b}+m_{b^{\prime}}} .
\end{gathered}
$$

Assuming also that constituents $a$ and $b$ are on mass shell, their momenta are [2]:

$$
\left(\begin{array}{l}
a_{0} \\
a_{z}
\end{array}\right)=\frac{1}{2}\left[x_{+}\left(A_{0}+A_{z}\right) \pm \frac{m_{a}^{2}}{\left(A_{0}+A_{z}\right)}\right]
$$

and

$$
\left(\begin{array}{c}
b_{0} \\
-b_{z}
\end{array}\right)=\frac{1}{2}\left[x_{-}\left(B_{0}-B_{z}\right) \pm \frac{m_{b}^{2}}{\left(B_{0}-B_{z}\right)}\right],
$$

and the invariant mass of $a$ and $b$ is then given by

$$
s_{a b}=(a+b)^{2}=\left(a_{0}+b_{0}\right)^{2}-\left(a_{z}+b_{z}\right)^{2} .
$$

We identify this with the argument $Q^{2}$ of the running coupling constant $\alpha_{s}\left(Q^{2}\right)$ in Eq. (74).

In Fig. 4 we show a test of the accuracy of this scattering model with experimental data in an analogous low-energy reaction, $I=2 \pi \pi$ scattering. The prediction for the dominant $S$-wave phase shift is shown together with the data of Hoogland et al. [41]. Note that this is not a fit; all the parameters are determined by meson spectroscopy, which fixes the interquark forces and wavefunctions that are then used to calculate the meson-meson 
scattering amplitude.

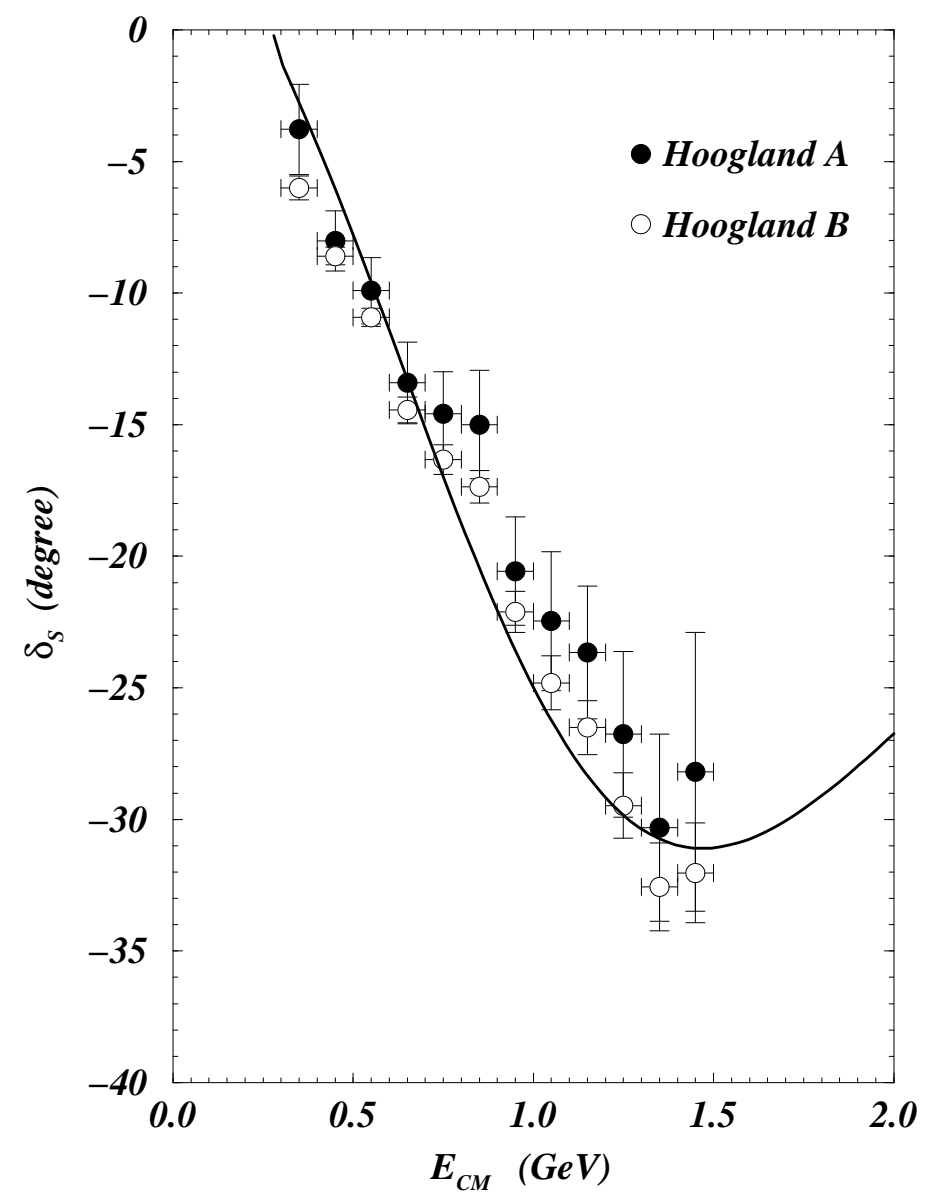

Fig. 4. Comparison of our theoretical $I=2 \pi \pi$ phase shift (solid curve) with the data of Hoogland et al. 41.

\section{A TEST OF 'POST-PRIOR' EQUIVALENCE IN $\pi+J / \psi$ DISSOCIATION}

Fig. 1 shows the 'prior' meson-meson scattering diagrams, in which the interactions occur before constituent interchange. There is a corresponding 'post' set of diagrams, in which the interactions occur after constituent interchange, as shown in Fig. 2. As we noted earlier, these are equivalent descriptions of the transition matrix element, and should give identical cross sections.

In nonrelativistic scattering theory the post and prior results can be formally proven to be equivalent, so that the two theoretical cross sections are indeed identical. This proof requires that the interaction $V$ used to determine the external meson wavefunctions be identical to the interaction used in the evaluation of the scattering amplitude. Numerical confirmation of this post-prior equivalence constitutes a very nontrivial test of the accuracy of the numerical procedures used both in determining the bound state wavefunction and in evaluating the complete meson-meson scattering amplitude. This post-prior equivalence was discussed in detail and demonstrated numerically by Swanson for $\pi \pi \rightarrow \rho \rho$ scattering 
in Ref. [34, where its relevance to establishing Hermitian effective scattering potentials was shown.

To test post-prior equivalence in our $J / \psi$ dissociation calculations (in the nonrelativistic formalism), we have carried out the evaluation of the cross section using both post and prior formalisms. Of necessity we assumed nonrelativistic kinematics and theoretical masses to determine the external meson momenta $|\boldsymbol{A}|$ and $|\boldsymbol{C}|$, which appear in the overlap integrals. The post-prior equivalence holds if the coupling constant does not depend on energy. We are well advised to use a set of parameters with a fixed running coupling constant $\alpha_{s}$ for this test. We used therefore the parameter set [21] $\alpha_{s}=0.58, b=0.18 \mathrm{GeV}^{2}, \sigma=0.897 \mathrm{GeV}$, $m_{u, d}=0.345 \mathrm{GeV}, m_{c}=1.931 \mathrm{GeV}$, and $V_{\text {con }}=-0.612 \mathrm{GeV}$, which are close to standard values and were chosen because they reproduce the physical masses of the $\pi, D, D^{*}, J / \psi$ and $\psi^{\prime}$ rather well. Fig. $5 a$ shows the dissociation cross sections for $\pi+\psi$ collisions as a function of the initial kinetic energy $E_{K E}$ measured in the center-of-mass frame, defined as $E_{K E}=$ $\sqrt{s}-m_{A}-m_{B}$ where $m_{A}$ and $m_{B}$ are the masses of the incident mesons. The differences between the post and prior results in Fig. $5 a$ are indeed rather small, which confirms postprior equivalence assuming non-relativistic dynamics. (The residual discrepancy is mainly due to our use of a truncated basis in expanding the meson wavefunctions.) Fig. $5 b$ shows the corresponding results for $\pi+\psi^{\prime}$ dissociation. In this case there is greater sensitivity to the approximate $\psi^{\prime}$ wavefunction, due to large cancellations in the numerical integration of a radially-excited wavefunction.

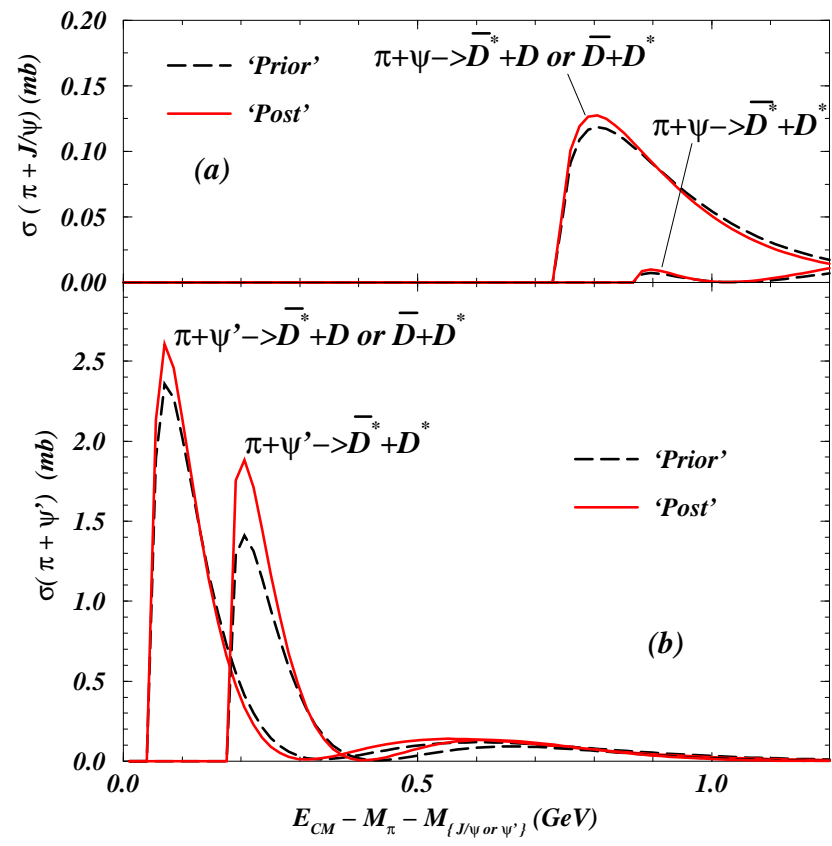

Fig. 5. Comparison of $\pi+J / \psi$ and $\pi+\psi^{\prime}$ cross sections $=$ derived using post and prior formalisms.

In the comparisons shown in Fig. 5 we have used theoretical masses for the mesons; the proof of post-prior equivalence makes use of the theoretical bound-state masses from the Schrödinger equation with the given potential, rather than the experimental ones. Our theoretical masses are close to experiment but are not exact, as is evident in Fig. 3 . If 
one instead assumes experimental values for the meson masses, there will be additional post-prior discrepancies in our cross section calculations. In our subsequent cross section calculations we do assume experimental masses in order to reproduce correct thresholds; the consequence is a systematic uncertainty in the cross sections which may be estimated by comparing post and prior predictions.

It should be noted that 'post-prior equivalence' had only been proven for bound-state scattering in non-relativistic quantum mechanics [39]. Recently an extension of this proof to scattering in a relativistic generalization of quantum mechanics was given by Wong and Crater [42], using Dirac's constraint dynamics. A full relativistic treatment of this problem will involve the derivation of relativistic two-body wavefunctions and Wigner spin rotations, which is beyond the scope of the present investigation. We will adopt an intermediate approach and assume relativistic kinematics for the initial and final mesons and use relativistic phase space; in consequence we find different 'post' and 'prior' cross sections in general. Here we will take the mean value of the 'post' and 'prior' results as our estimated cross section, separate 'post' and 'prior' cross sections will be shown as an indication of our systematic uncertainty.

\section{CROSS SECTIONS FOR $J / \psi$ AND $\psi^{\prime}$ DISSOCIATION}

Depending on the incident energies, dissociation of the $J / \psi$ and $\psi^{\prime}$ by pions can lead to many different exclusive final states. There are several selection rules that eliminate or suppress many of the a priori possible final channels, given our simple quark-model Hamiltonian and Born-order scattering amplitudes. Considerable simplification follows from the fact that our model Hamiltonian conserves total spin $S_{t o t}$. Since the $J / \psi$ and $\psi^{\prime}$ have $S=1$ and pions have $S=0$, the initial and final states in $\pi+J / \psi$ and $\pi+\psi^{\prime}$ collisions must both have $S_{\text {tot }}=1$; this forbids $D \bar{D}$ final states. With increasing invariant mass we next encounter the final states $D \bar{D}^{*}, D^{*} \bar{D}$ and $D^{*} \bar{D}^{*}$. In Fig. 6 we show the exclusive dissociation cross sections for $\pi+J / \psi$ and $\pi+\psi^{\prime}$ collisions into these first few allowed final states. The total dissociation cross section, which is the sum of the exclusive cross sections, is shown as a solid line. Our estimate is the mean of the total 'post' and 'prior' cross sections, which are also shown in Fig. 6. The estimated range of uncertainty, due to the post-prior discrepancy and to parameter variations, is shown as a shaded band.

The $J / \psi$ dissociation processes $\pi+J / \psi \rightarrow D^{*} \bar{D}$ or $D \bar{D}^{*}$ have a threshold of about $E_{K E}=0.65 \mathrm{GeV}$ and the total dissociation cross section reaches approximately $1 \mathrm{mb}$ not far above threshold (Fig. 6a). This $\pi+J / \psi$ cross section is considerably smaller than the peak value of about $7 \mathrm{mb}$ found by Martins et al., largely due to their assumption of a color-independent confining interaction.

The threshold for $\pi+\psi^{\prime} \rightarrow D \bar{D}^{*}+D^{*} \bar{D}$ is only about $E_{K E}=0.05 \mathrm{GeV}$, and in consequence the $\psi^{\prime}$ cross sections are much larger near threshold. The total $\pi+\psi^{\prime}$ dissociation cross section has maxima of $\approx 4.2(0.5) \mathrm{mb}$ and $\approx 2.8(0.5) \mathrm{mb}$ at $E_{K E} \approx 0.1 \mathrm{GeV}$ and $\approx 0.22$ $\mathrm{GeV}$ respectively (Fig. $6 b$ ). It is interesting that the exclusive $\psi^{\prime}$ dissociation cross sections are very small near $E_{K E}=0.3$ (for the $D \bar{D}^{*}$ final state) and $0.4 \mathrm{GeV}$ (for the $D^{*} \bar{D}^{*}$ final 
state), due to almost complete destructive interference between the diagrams.

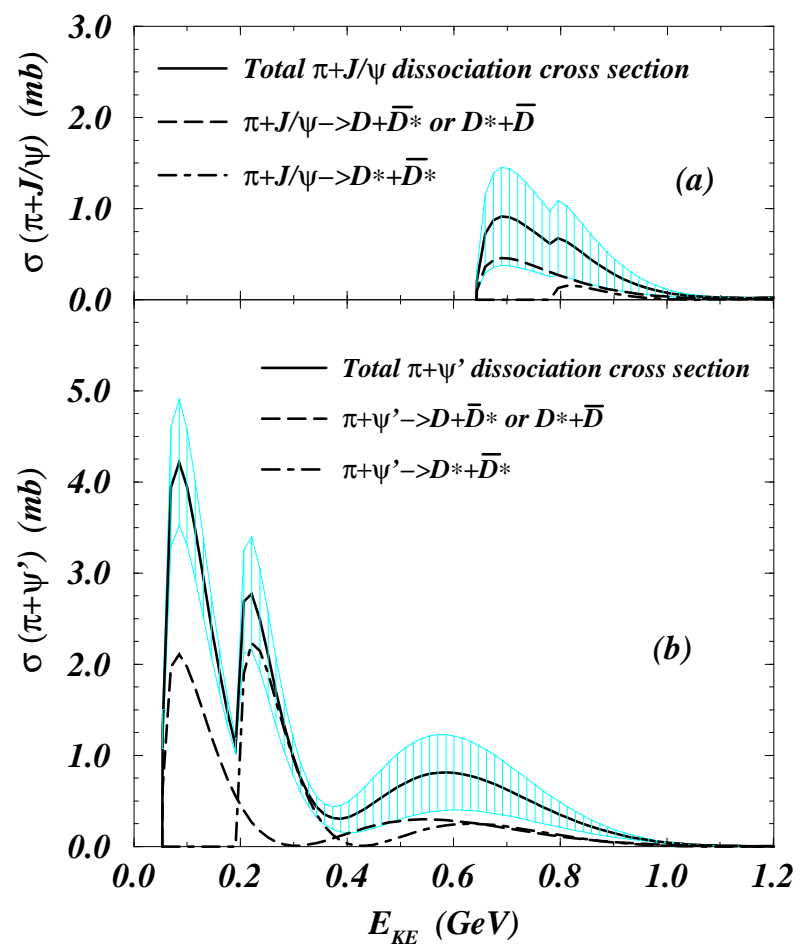

Fig. 6. Exclusive and total dissociation cross sections for $\pi+J / \psi$ (Fig. 6a) and $\pi+\psi^{\prime}$ (Fig. 6b). In each panel the solid curve gives our estimated total cross section, which is the mean of the 'prior' and 'post' results.

The relative importance of the various terms in the Hamiltonian in these dissociation amplitudes is of course a very interesting question. Unfortunately it is also somewhat ambiguous, because the individual terms differ between post and prior formalisms; only the sum of all terms is formalism independent in non-relativistic quantum mechanics. We find that the spin-spin interaction makes the dominant contribution to $\pi+J / \psi$ dissociation in the prior formalism; $\pi+\psi^{\prime}$ dissociation in contrast is dominated by the linear confining interaction. In the post formalism we find that both $\pi+J / \psi$ and $\pi+\psi^{\prime}$ are dominated by the spin-spin interaction. In all these cases the color-Coulomb contribution is subdominant.

Our results have interesting consequences for the survival of $J / \psi$ and $\psi^{\prime}$ mesons propagating in a gas of pions. The pions produced in a heavy ion collision have a roughly thermal distribution, with $T \approx 200 \mathrm{MeV}$ in the nucleus-nucleus center-of-mass system, whereas heavy quarkonia such as the $J / \psi$ and $\psi^{\prime}$ are produced approximately at rest. Thus the relative kinetic energy is typically a few hundred $\mathrm{MeV}$. This is below the $\pi+J / \psi$ dissociation threshold, but above that of $\pi+\psi^{\prime}$, and in consequence we expect $\pi+\psi^{\prime}$ collisions to deplete the $\psi^{\prime}$ population much more effectively than $\pi+J / \psi$ collisions reduce the initial $J / \psi$ population. The weakness of $\pi+J / \psi$ dissociation is further reduced by the small cross section we find for the $\pi+J / \psi$ relative to $\pi+\psi^{\prime}$.

Next we consider the very interesting exothermic collisions of charmonia with light vector mesons, specifically $\rho+J / \psi$ and $\rho+\psi^{\prime}$. Since the $\rho$ meson has $S=1$, the total spin of the 
$\rho+\left\{J / \psi\right.$ or $\left.\psi^{\prime}\right\}$ system can be $S_{t o t}=\{0,1$, and 2$\}$. This $S_{\text {tot }}$ is conserved in our model, and so must agree with the $S_{\text {tot }}$ of the final state. The low-lying open charm final states are $D \bar{D}$ with $S_{t o t}=0, D \bar{D}^{*}$ and $D^{*} \bar{D}$ with $S_{t o t}=1$, and $D^{*} \bar{D}^{*}$ with $S_{t o t}=\{0,1$, and 2$\}$. The contribution of transitions to radially- and orbitally-excited final states will be considered in future work 43.

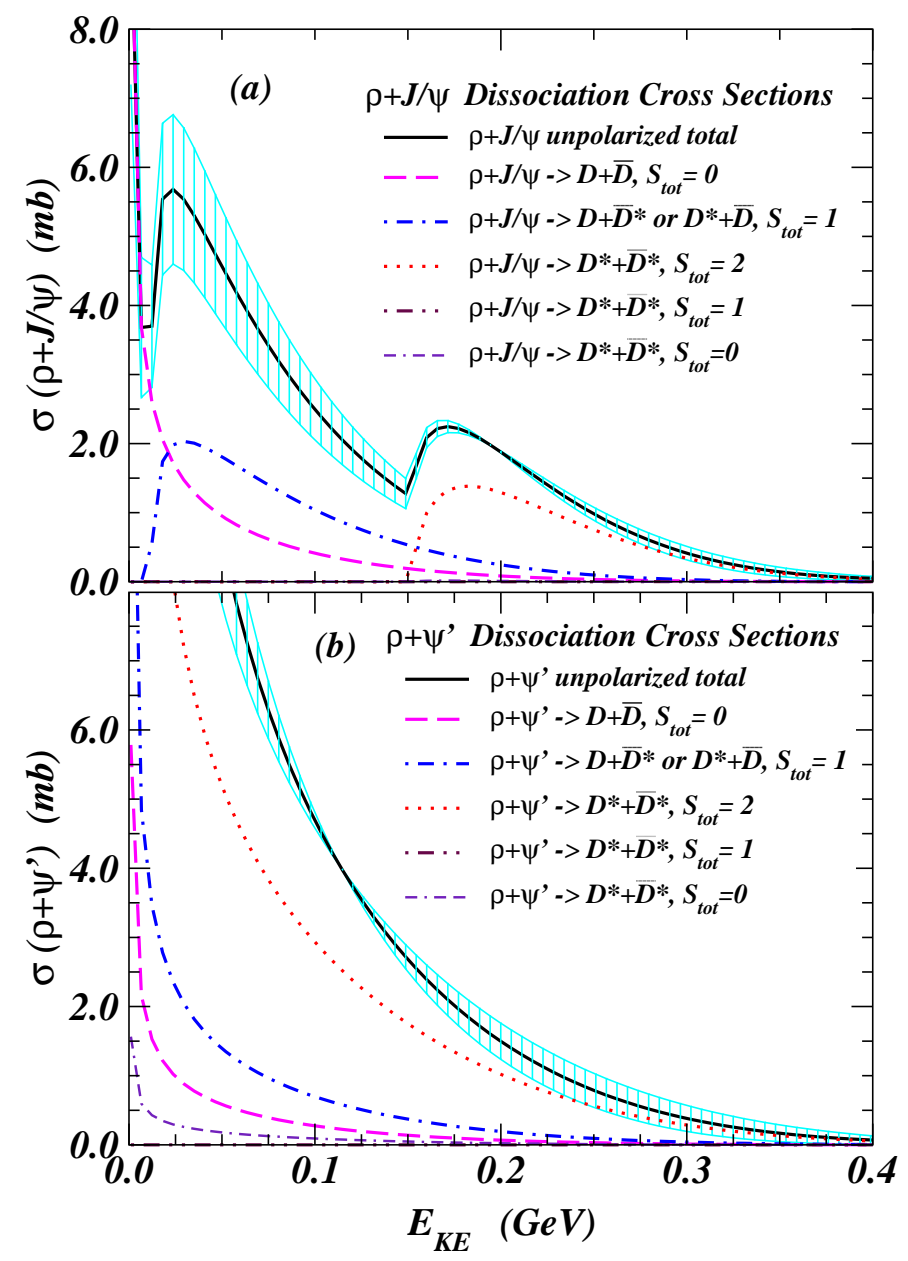

Fig. 7. The total unpolarized dissociation cross sections are shown as the solid curves for $\rho+J / \psi$ in Fig. $7 a$ and for $\rho+\psi^{\prime}$ in Fig. 7b. Unpolarized exclusive dissociation cross sections $\sigma_{f}^{\text {unpol }}\left(S_{t o t}\right)$ for different final states and different $S_{\text {tot }}$ are also shown.

In the collision of an unpolarized $\rho$ with an unpolarized $J / \psi$, the total dissociation cross section comprises of contributions $\sigma_{f}^{\text {unpol }}\left(S_{t o t}\right)$ from different final states $f$ and different total spin values $S_{\text {tot }}$ of the system,

$$
\sigma_{\text {tot }}^{\mathrm{unpol}}=\sum_{f} \sum_{S_{t o t}} \sigma_{f}^{\mathrm{unpol}}\left(S_{t o t}\right)
$$

where for this case with $L_{A}=0$ and $S_{B}=S_{\rho} \neq 0$ we can deduce from Eq. (17) the following relationship 


$$
\sigma_{f}^{\mathrm{unpol}}\left(S_{t o t}\right)=\frac{\left(2 S_{t o t}+1\right)}{\left(2 S_{\rho}+1\right)\left(2 S_{J / \psi}+1\right)} \sigma_{f}\left(S_{t o t}\right),
$$

and $\sigma_{f}\left(S_{t o t}\right)$ is the dissociation cross section for the final state $f$ when the initial twomeson system is prepared with a total spin $S_{\text {tot }}$. [In our earlier work [2] for $\rho+J / \psi$ and $\rho+\psi^{\prime}$ collisions, $\sigma_{f}\left(S_{t o t}\right)$ results were presented and the total cross section of $\sigma_{\text {tot }}=$ $\sum_{f} \sum_{S_{t o t}} \sigma\left(S_{t o t}\right)$ was evaluated. However, for the collision of unpolarized mesons, one should use the unpolarized total dissociation cross section as given by Eqs. (80) and (81).].

The unpolarized total $\rho+J / \psi$ dissociation cross section are shown in Fig. 7a. The exclusive cross sections $\sigma_{f}^{\text {unpol }}\left(S_{t o t}\right)$ for dissociating into various final states in an unpolarized collision are also exhibited. The reaction $\rho+J / \psi \rightarrow D \bar{D}$ is exothermic, so the cross section $\sigma_{D \bar{D}}^{\text {unpol }}\left(S_{\text {tot }}=0\right)$ diverges as $1 / \sqrt{E_{K E}}$ as we approach threshold. For other $\rho+J / \psi$ exclusive final states the thresholds lie at sufficiently higher energies to be endothermic. We find an unpolarized total $\rho+J / \psi$ dissociation cross section of $2.4(0.4)$ mb at $E_{K E}=0.1 \mathrm{GeV}$, which has decreased to about $1.9 \mathrm{mb}$ at $E_{K E}=0.2 \mathrm{GeV}$. At very low kinetic energies, the contribution to the dissociation of $J / \psi$ by $\rho$ comes from the $D \bar{D}\left(S_{t o t}=0\right)$ final state. At slightly higher energy, it comes mainly from $D \bar{D}^{*}$ and $D^{*} \bar{D}\left(S_{t o t}=1\right)$ final states. At $E_{K E}$ about $0.2 \mathrm{GeV}$, it comes dominantly from the $D^{*} \bar{D}^{*}\left(S_{t o t}=2\right)$ final state.

We have carried out similar calculations for $\rho+\psi^{\prime}$ collisions, and the results are shown in Fig. 7b. In this case the reactions $\rho+\psi^{\prime} \rightarrow D \bar{D}, D \bar{D}^{*}, D^{*} \bar{D}$ and $D^{*} \bar{D}^{*}$ are all exothermic, so all these exclusive cross sections $\sigma_{f}^{\text {unpol }}\left(S_{t o t}\right)$ diverge as $1 / \sqrt{E_{K E}}$ as we approach threshold. The dominant contribution to the dissociation comes from the $D^{*} \bar{D}^{*}\left(S_{t o t}=2\right)$ final state. The total unpolarized $\rho+\psi^{\prime}$ dissociation cross section falls from $4.5(0.1) \mathrm{mb}$ at $E_{K E}=$ $0.1 \mathrm{GeV}$ to $1.5(0.3) \mathrm{mb}$ at $E_{K E}=0.2 \mathrm{GeV}$ and $0.4(0.2) \mathrm{mb}$ at $E_{K E}=0.3 \mathrm{GeV}$.

In $\rho+J / \psi$ dissociation the dominant scattering contribution in the prior formalism is due to the linear interaction. In the post formalism the dominant contribution arises from the color-Coulomb and linear interactions at energies $E_{K E}<0.1 \mathrm{GeV}$, from color-Coulomb at $0.1 \mathrm{GeV}<E_{K E}<0.4 \mathrm{GeV}$, and from the spin-spin interaction at $E_{K E}>0.4 \mathrm{GeV}$.

We turn next to the dissociation of $J / \psi$ and $\psi^{\prime}$ in collision with $K$. Our predictions for $K+J / \psi$ and $K+\psi^{\prime}$ dissociation cross sections are shown in Fig. 8. The $K+J / \psi$ process has a threshold kinetic energy of about $0.4 \mathrm{GeV}$, and the maximum cross section is about $0.7 \mathrm{mb}$. In $K+\psi^{\prime}$ dissociation the reactions are exothermic for the allowed final states $D_{s}+\bar{D}^{*}, D_{s}^{*}+\bar{D}$ and $D_{s}^{*}+\bar{D}^{*}$. The total $K+\psi^{\prime}$ dissociation cross section, shown in Fig. $8 b$, 
is about $1 \mathrm{mb}$ at $E_{K E} \sim 0.4 \mathrm{GeV}$, and also diverges as $1 / \sqrt{E_{K E}}$ as we approach threshold.

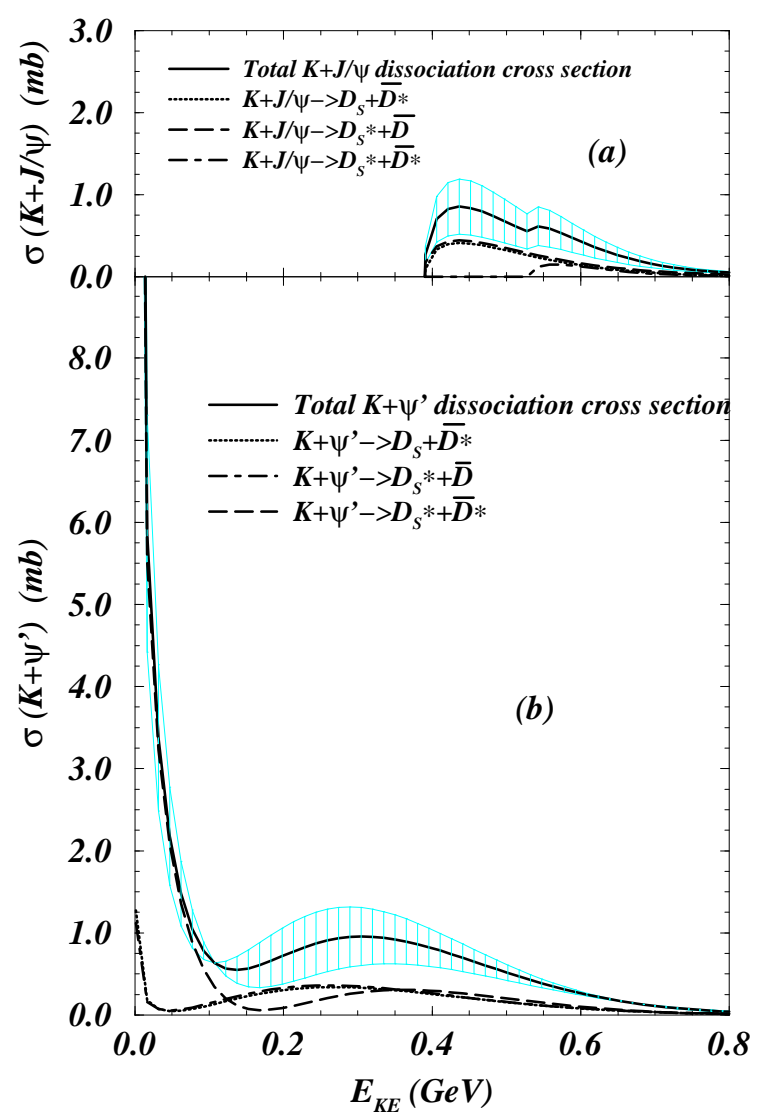

Fig. 8. Dissociation cross sections for $K+J / \psi$ (Fig. $8 a$ ) and $K+\psi^{\prime}$ (Fig. 8b).

The model of Barnes and Swanson has thus far received experimental support from extensive comparisons with light hadron scattering data $(I=2 \pi \pi$ 33., $I=3 / 2 K \pi$, 35 , $I=0,1 K N$ [36]). The new results of $J / \psi$ and $\psi^{\prime}$ dissociation in collision with $\pi, \rho$, and $K$ need to confront experimental data through an examination of their implications on $J / \psi$ and $\psi^{\prime}$ suppression in high-energy heavy-ion collisions. The extent to which the observed anomalous suppression in $\mathrm{Pb}+\mathrm{Pb}$ collisions is due to the dissociation $J / \psi$ by $\pi, \rho$, and $K$ will require further quantitative study. $J / \psi$ suppression by these mesons must be incorporated and subtracted in order to identify the suppression of $J / \psi$ production by the quark-gluon plasma. It is therefore important to carry out a detailed simulation of $J / \psi$ absorption using cross sections obtained here in order to understand the nature of $J / \psi$ suppression in $\mathrm{Pb}+\mathrm{Pb}$ collisions and to provide a test of the theoretical $J / \psi$ dissociation cross sections.

\section{CROSS SECTIONS FOR $\Upsilon$ AND $\Upsilon^{\prime}$ DISSOCIATION.}

It has been noted that the suppression of the production rates of $b \bar{b}$ mesons, such as the $\Upsilon$ and $\Upsilon^{\prime}$, may also be useful as a signal for QGP production (see Ref. 32 and references cited therein). It is of interest to calculate the $\Upsilon$ and $\Upsilon^{\prime}$ dissociation cross section in collisions 
with $\pi, \rho$, and $K$ so as to infer the effects of $\Upsilon$ and $\Upsilon^{\prime}$ suppression by hadronic produced particles.
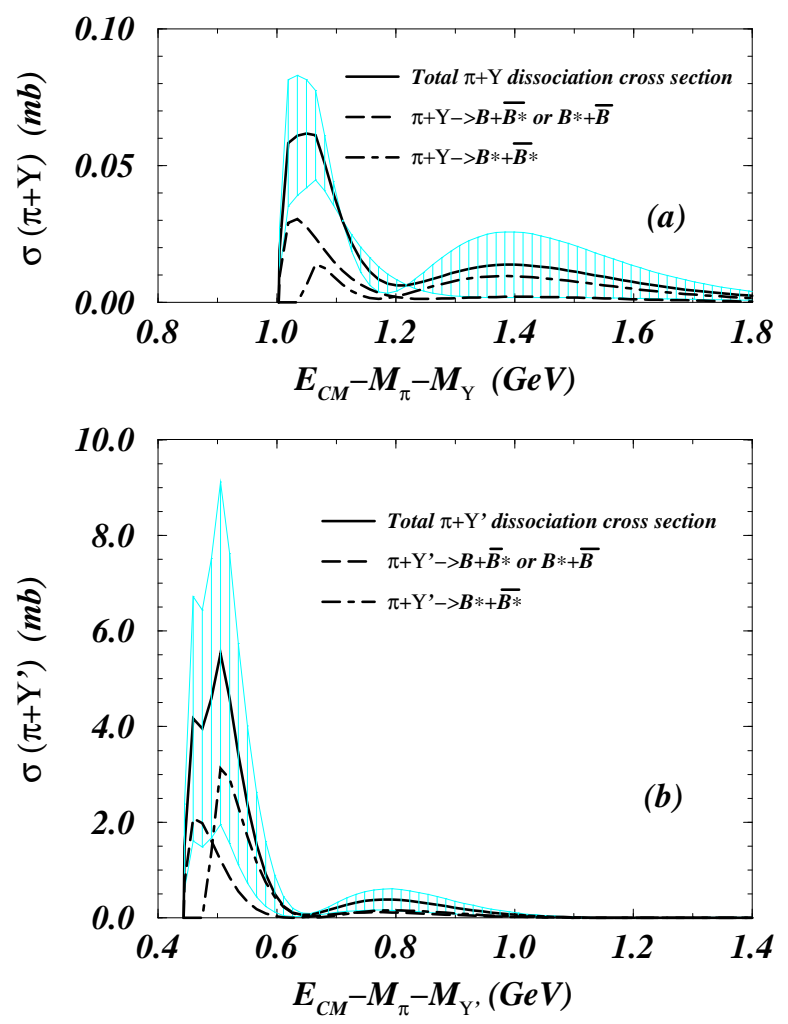

Fig. 9. The dissociation cross sections for $\Upsilon$ (Fig. 9a) and $\Upsilon^{\prime}$ (Fig. $9 b$ ) in collision with $\pi$. Note that the scales of Figs. $9 a$ and $9 b$ are different.

We show in Fig. 9a the total cross section for the dissociation of the $\Upsilon$ in collision with $\pi$. The threshold is at $E_{K E} \sim 1 \mathrm{GeV}$, and the maximum cross section is about $0.05 \mathrm{mb}$. The small cross section arises from the combined effects of a large threshold and a small value of the strong interaction coupling constant. We show the dissociation cross section for $\Upsilon^{\prime}$ in collision with $\pi$ in Fig. 9b. It has a threshold of $E_{K E} \sim 0.45 \mathrm{GeV}$, and the peak cross section is about $5 \mathrm{mb}$ at $E_{K E} \sim 0.55 \mathrm{GeV}$. This dissociation cross section is much larger than that for the dissociation of $\Upsilon$ by $\pi$.

In Fig. $10 a$ we show the unpolarized cross section for the dissociation of $\Upsilon$ in collision with $\rho$. Unpolarized exclusive dissociation cross sections $\sigma_{f}^{\text {unpol }}\left(S_{\text {tot }}\right)$ for different final states and different $S_{\text {tot }}$ are also shown. The reaction process is endothermic with a threshold at $E_{K E} \sim 0.3 \mathrm{GeV}$ and a peak cross section of $0.15 \mathrm{mb}$ at $E_{K E} \sim 0.45 \mathrm{GeV}$. In Fig. $10 b$ we show the dissociation cross section for $\Upsilon^{\prime}$ in collision with $\rho$. The reactions are exothermic and the total dissociation cross section behaves as $1 / \sqrt{E_{K E}}$ near $E_{K E} \sim 0$ and decreases to 
about $1.6 \mathrm{mb}$ at $E_{K E} \sim 0.2 \mathrm{GeV}$.
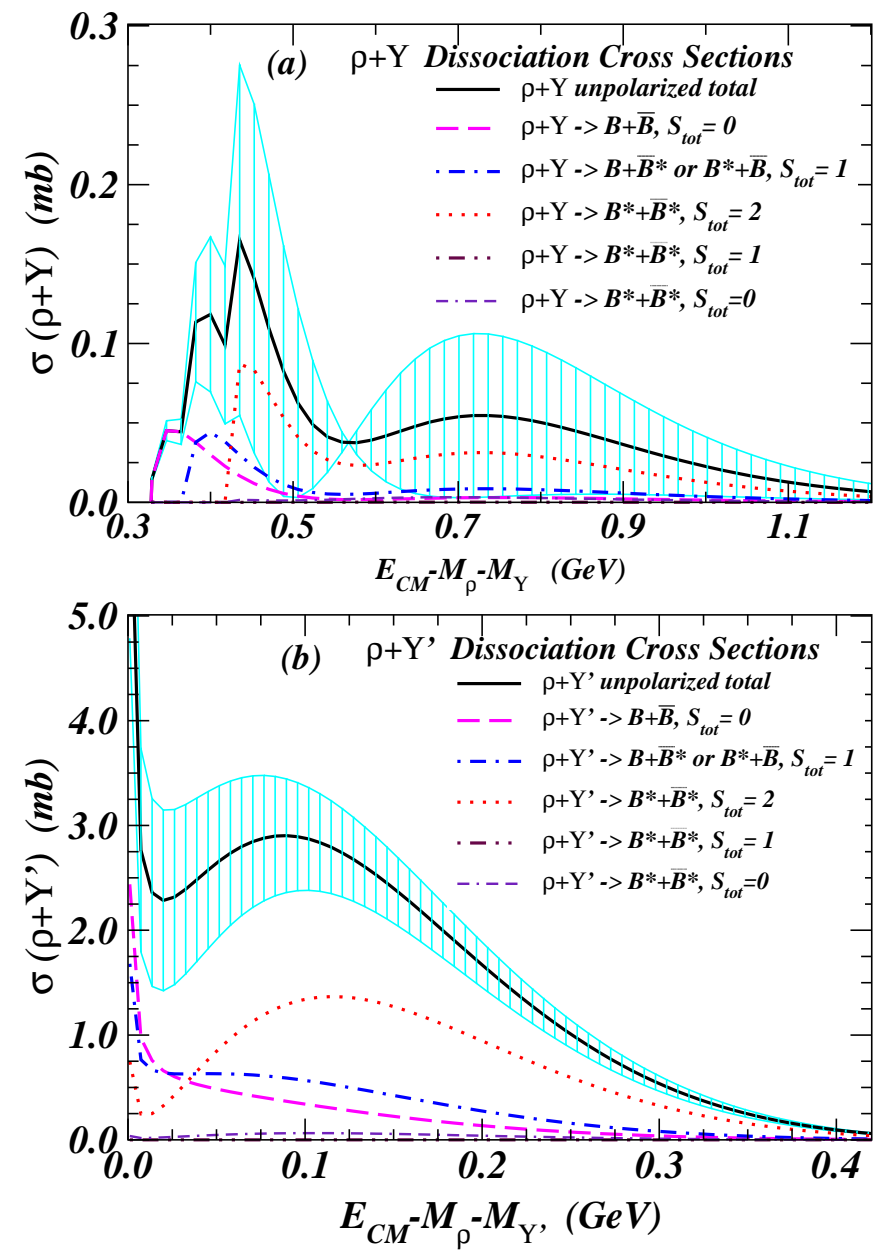

Fig. 10. Unpolarized total dissociation cross sections and unpolarized exclusive dissociation cross sections $\sigma_{f}^{\text {unpol }}\left(S_{t o t}\right)$ for $\rho+\Upsilon$ (Fig. $10 a)$ and $\rho+\Upsilon^{\prime}$ (Fig. 10b) for various channels and total spin $S_{t o t}$. Note that Figs. $10 a$ and $10 b$ have different scales.

In Fig. $11 a$ we show the cross section for the dissociation of $\Upsilon$ by $K$. The threshold is at $E_{K E} \sim 0.75 \mathrm{GeV}$, with a peak total cross section of about $0.05 \mathrm{mb}$ at $E_{K E} \sim 0.85 \mathrm{GeV}$. We show in Fig. $11 b$ the dissociation cross section of $\Upsilon^{\prime}$ by $K$. The threshold is at $E_{K E} \sim 0.2$ 
$\mathrm{GeV}$, with a peak total cross section of about $2 \mathrm{mb}$ at $E_{K E} \sim 0.25 \mathrm{GeV}$.
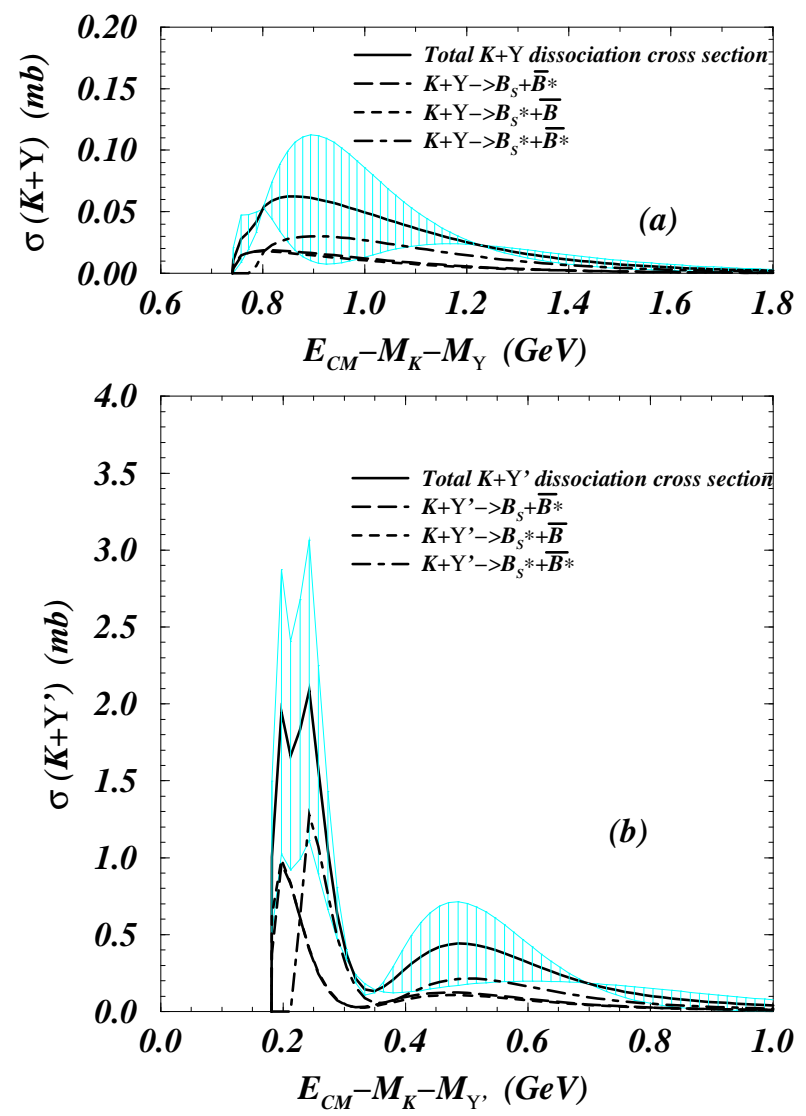

Fig. 11. The dissociation cross sections for $K+\Upsilon^{\prime}$ (Fig. 11a) and $K+\Upsilon^{\prime}$ (Fig. 11b) for various final states. Note that Figs. 11a and $11 b$ have different scales.

\section{CROSS SECTIONS FOR $\chi_{J}$ DISSOCIATION}

We can calculate the dissociation cross sections of $\chi_{J}$ mesons in collision with $\pi, \rho$, and $K$ using the quark-interchange model. A $\chi_{J}$ meson has a spin quantum number $S=1$ and a $\pi$ has $S=0$. The collision of a $\chi_{J}$ with a $\pi$ gives rise to a system with a total spin $S_{t o t}=1$. On the other hand, the interaction of Eq. (2), which leads to the dissociation, conserves the total spin. Therefore the lowest-energy final states are $D \bar{D}^{*}, D^{*} \bar{D}$, and $D^{*} \bar{D}^{*}$.

We show the results for the dissociation cross sections of unpolarized $\chi_{J}$ in collision with $\pi$ in Fig. 12. The unpolarized dissociation cross sections for the final states of $D \bar{D}^{*}$ and $D^{*} \bar{D}^{*}$ are shown as the dotted and the dashed-dot curves respectively. The unpolarized total cross sections for scattering into these lowest channels are shown as the solid curve obtained as the mean value of the total cross section from the 'prior' and 'post' formalisms. The estimated systematic uncertainty in the total cross section due to the post-prior discrepancy is again indicated as a band in the figure. The dissociation of $\chi_{0}$ by $\pi$ has a threshold of $E_{K E} \sim 0.32 \mathrm{GeV}$ and a peak cross section of $1.53 \mathrm{mb}$ at $E_{K E} \sim 0.5 \mathrm{GeV}$ (Fig. 12a). The dissociation of $\chi_{1}$ by $\pi$ has a threshold of $E_{K E} \sim 0.23 \mathrm{GeV}$ and a peak dissociation cross section of $2.41 \mathrm{mb}$ at $E_{K E} \sim 0.46 \mathrm{GeV}$ (Fig. 12b). The dissociation of $\chi_{2}$ by $\pi$ has 
a threshold of $E_{K E} \sim 0.18 \mathrm{GeV}$ and a peak dissociation cross section of about $2.98 \mathrm{mb}$ at $E_{K E} \sim 0.41 \mathrm{GeV}$ (Fig. 12c).

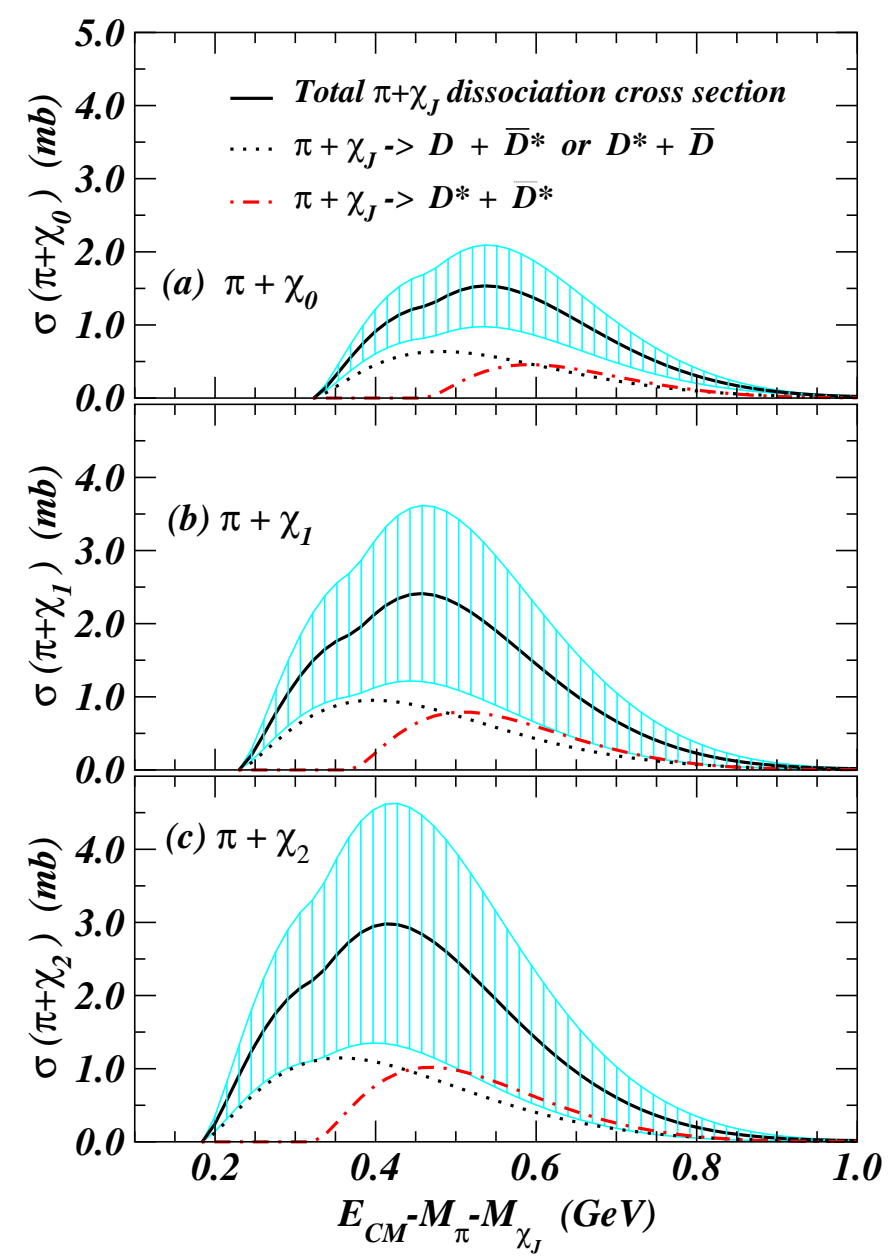

Fig. 12. Unpolarized total cross sections and exclusive unpolarized cross sections for the dissociation of $\chi_{J}$ into $D+\bar{D}^{*}$ and $D^{*}+\bar{D}^{*}$ in collision with $\pi$. Fig. $12 a$ is for $\pi+\chi_{0}$, Fig. $12 b$ for $\pi+\chi_{1}$, and Fig. $12 c$ for $\pi+\chi_{2}$.

It is interesting to note that the maximum of the unpolarized total dissociation cross section for $\pi+\chi_{2}$ is only slightly greater than that for $\pi+\chi_{1}$ but is nearly twice as great as the maximum of the dissociation cross section for $\pi+\chi_{0}$. This indicates that the dissociation of $\chi_{J}$ is very sensitive to the threshold value. We found numerically that if the threshold value for $\pi+\chi_{0}$ were taken to be the same as the threshold value for $\pi+\chi_{2}$, the unpolarized dissociation cross sections would be the same.

The dissociation amplitudes of the $\chi_{J J_{z}}$ mesons in collision with pions depend on $J_{z}$. A detailed discussion of the dissociation cross section for various $J$ and $J_{z}$ substates will be presented in [43]. The dependence on $J_{z}$ is however quite weak. For the same value of $J$, dissociation cross sections of $\chi_{J_{z}}$ in collision with $\pi$ vary only by a few percent for different 
$J_{z}$

The thresholds for $\pi+\chi_{J}$ dissociation lie in between those of $\pi+J / \psi$ and $\pi+\psi^{\prime}$, and the maxima of the total dissociation cross sections for the $\pi+\chi_{J}$ collisions are greater than that for the $\pi+J / \psi$ collision but less than for the $\pi+\psi^{\prime}$ collision.

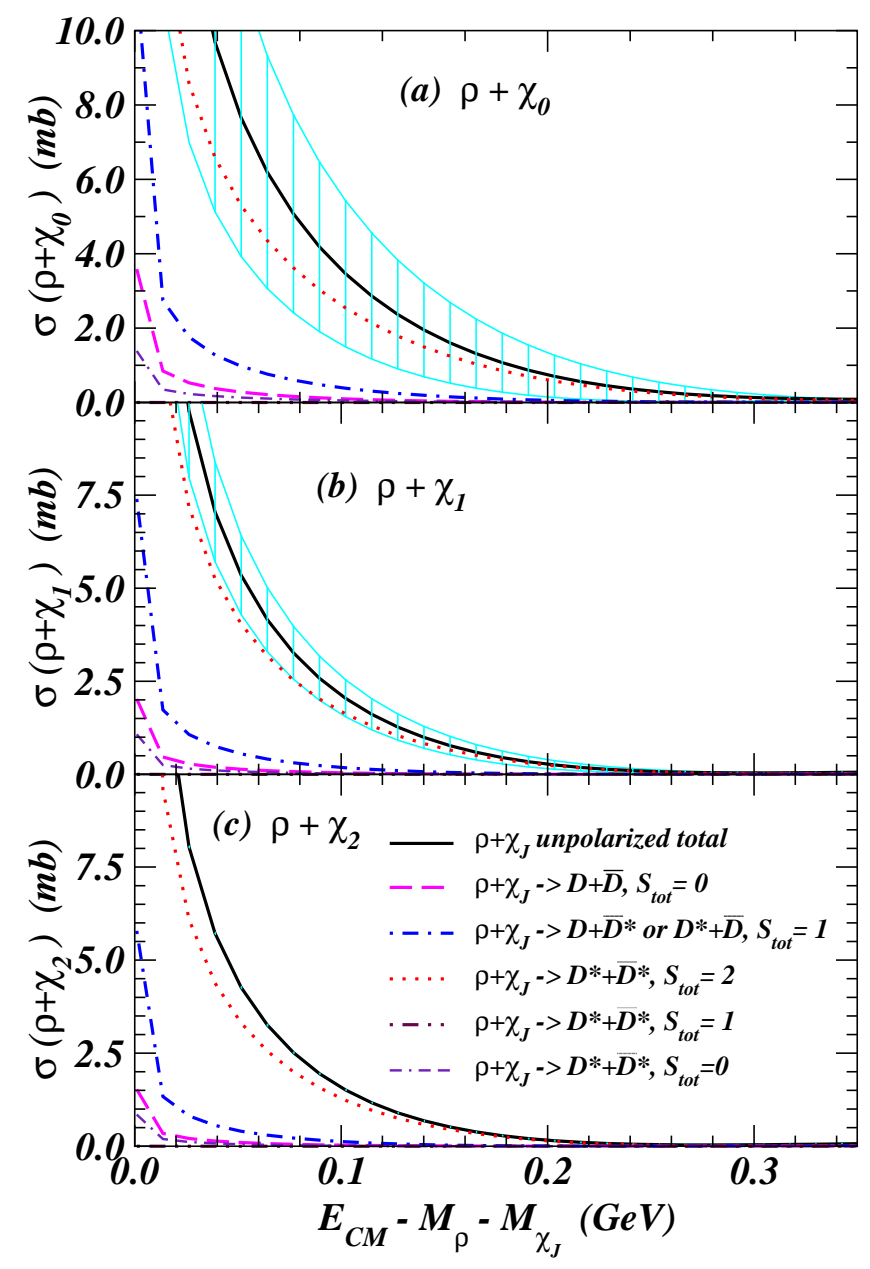

Fig. 13. Unpolarized total cross sections and unpolarized exclusive cross sections $\sigma_{f}^{\text {unpol }}\left(S_{t o t}\right)$ for the dissociation of $\chi_{J}$ into $D \bar{D}, D+\bar{D}^{*}$ and $D^{*}+\bar{D}^{*}$ in collision with $\rho$. Fig. $13 a$ is for $\rho+\chi_{0}$, Fig. $13 b$ for $\rho+\chi_{1}$, and Fig. $13 c$ for $\rho+\chi_{2}$.

We show in Fig. 13 the dissociation cross sections of unpolarized $\chi_{J}$ in collision with unpolarized $\rho$. The lowest-energy final states are $D \bar{D}, D \bar{D}^{*}, D^{*} \bar{D}$, and $D^{*} \bar{D}^{*}$, characterized by different values of the total spin $S_{t o t}$. The unpolarized total dissociation cross section comprises of contribution $\sigma_{f}^{\text {unpol }}\left(S_{\text {tot }}\right)$ from different final states $\{f\}$ and different total spins $\left\{S_{t o t}\right\}$ of the system,

$$
\sigma_{\text {tot }}^{\text {unpol }}=\sum_{f} \sum_{S_{t o t}} \sigma_{f}^{\mathrm{unpol}}\left(S_{t o t}\right)
$$

where for this case with $L_{A}=1$ and $S_{B}=1, \sigma_{f}^{\text {unpol }}\left(S_{t o t}\right)$ is more complicated than the 
expression of Eq. (81) for $L_{A}=0$ and $S_{B}=1$. It can be determined from Eq. (15) and is given by

$$
\sigma_{f}^{\text {unpol }}\left(S_{t o t}\right)=\sum_{J M_{A}}(\hat{S} \hat{J})^{2}\left\{\begin{array}{ccc}
S_{A} & S_{B} & S \\
L_{A} & 0 & L_{A} \\
J_{A} & S_{B} & J
\end{array}\right\}^{2} \sigma\left(L_{A} M_{A} S S_{z}\right),
$$

where $\sigma\left(L_{A} M_{A} S S_{z}\right)$ is the cross section for the initial meson system to have a total internal orbital angular momentum $L_{A}$ with azimuthal components $M_{A}$ and total spin $S$. We show in Fig. 13 the unpolarized total dissociation cross section for $\rho+\chi_{0}$ in Fig. $13 a, \rho+\chi_{1}$ in Fig. 13b, and $\rho+\chi_{2}$ in Fig. 13c. The exclusive cross section $\sigma_{f}^{\text {unpol }}\left(S_{t o t}\right)$ for different final states and $S_{t o t}$ are also shown. The dissociation of $\chi_{J}$ in collision with $\rho$ is exothermic. The dissociation cross sections have the common features that they diverge as $1 / \sqrt{E_{K E}}$ near $E_{K E} \sim 0$ and decreases monotonically as $E_{K E}$ increases. The dominant contribution to the dissociation cross sections comes from the $D^{*} \bar{D}^{*}\left(S_{t o t}=2\right)$ final state. The unpolarized total dissociation cross section for $\rho+\chi_{0}$ at $E_{K E}=0.05,0.1$, and $0.15 \mathrm{GeV}$ are $8.0 \mathrm{mb}, 3.5$ $\mathrm{mb}$, and $1.6 \mathrm{mb}$ respectively (Fig. 13a). The unpolarized total dissociation cross section for $\rho+\chi_{1}$ at $E_{K E}=0.05,0.1$, and $0.15 \mathrm{GeV}$ are $5.5 \mathrm{mb}, 2.0 \mathrm{mb}$, and $0.8 \mathrm{mb}$ respectively (Fig. $13 b)$. The unpolarized total dissociation cross section for $\rho+\chi_{2}$ at $E_{K E}=0.05,0.1$, and $0.15 \mathrm{GeV}$ are $4.3 \mathrm{mb}, 1.5 \mathrm{mb}$, and $0.5 \mathrm{mb}$ respectively (Fig. 13c). Thus, for the same kinetic energy $E_{K E}, \sigma_{t o t}^{\text {unpol }}\left(\rho+\chi_{0}\right)>\sigma_{\text {tot }}^{\text {unpol }}\left(\rho+\chi_{1}\right)>\sigma_{\text {tot }}^{\text {unpol }}\left(\rho+\chi_{2}\right)$.

We show in Fig. 14 the unpolarized dissociation cross section of $\chi_{J}$ in collision with $K$. The lowest-energy final states are $D_{s} \bar{D}^{*}, D_{s}^{*} \bar{D}$, and $D_{s}^{*} \bar{D}^{*}$. For the dissociation of $\chi_{0}$ in collision with $K$, the reaction has a threshold at $0.07 \mathrm{GeV}$. The total dissociation cross section rises from the threshold to a maximum cross section of $1.7 \mathrm{mb}$ at $0.27 \mathrm{GeV}$ (Fig. $14 a$ ). For the dissociation of $\chi_{1}$ and $\chi_{2}$ in collision with $K$, the reactions $K+\chi_{1}$ and $K+\chi_{2}$ are exothermic for the final states of $D_{s} \bar{D}^{*}$ and $D_{s}^{*} \bar{D}$. The total dissociation cross sections of $\chi_{1}$ and $\chi_{2}$ in collision with $K$ behave as $1 / \sqrt{E_{K E}}$ near $E_{K E} \sim 0$ but decrease very rapidly as $E_{K E}$ increases. These cross sections then maintain a relatively constant value up to 
$E_{K E} \sim 0.2 \mathrm{GeV}$ before decreasing at higher kinetic energies.

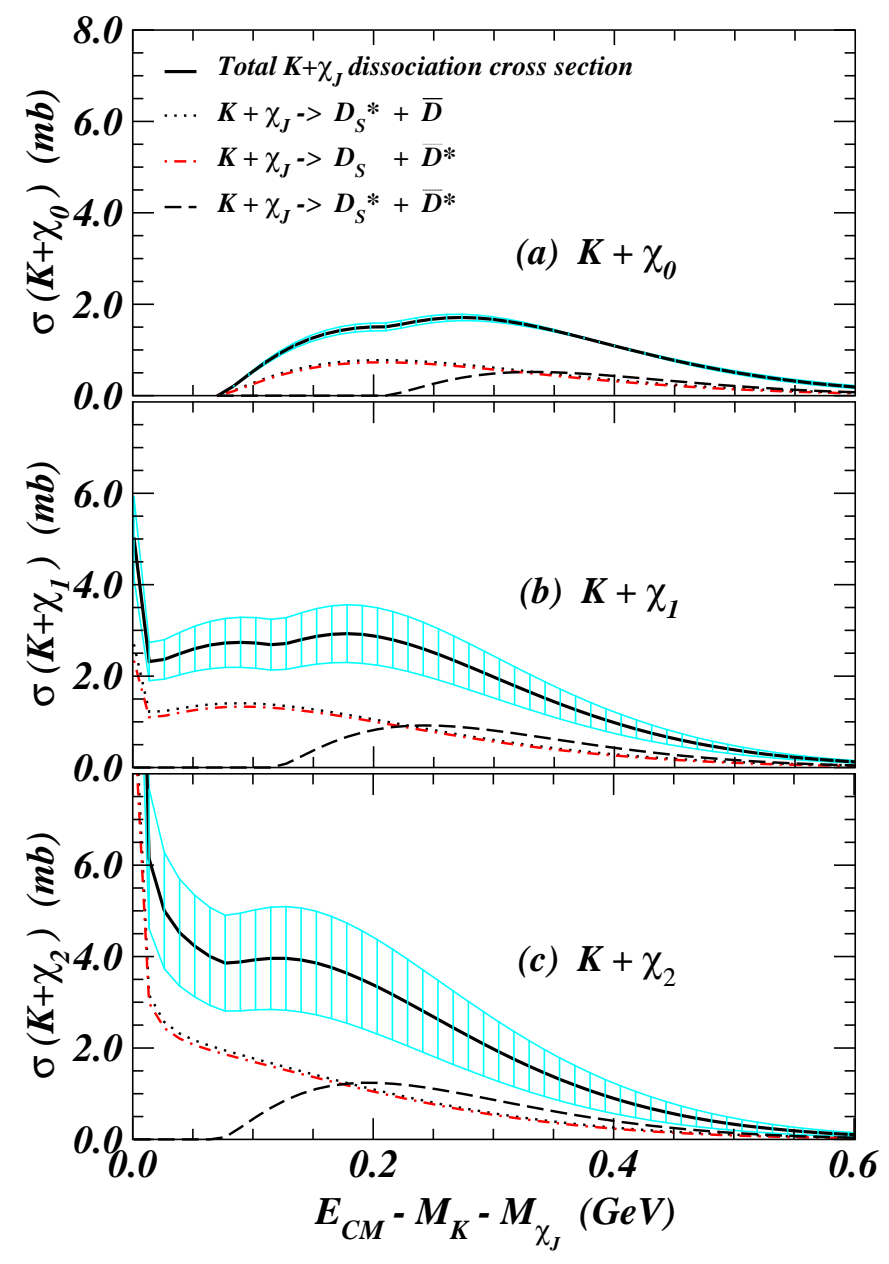

Fig. 14. Unpolarized cross sections for the dissociation of $\chi_{J}$ in collision with $K$. Fig. $14 a$ is for $K+\chi_{0}$, Fig. $12 b$ for $K+\chi_{1}$, and Fig. $14 c$ for $K+\chi_{2}$.

\section{DISCUSSION AND CONCLUSIONS}

We have used the Barnes-Swanson quark-interchange model, with parameters taken from fits to meson spectroscopy, to evaluate the low-energy cross sections for the dissociation of the $J / \psi, \psi^{\prime}, \chi, \Upsilon$, and $\Upsilon^{\prime}$ in collision with $\pi, \rho$ and $K$. The cross sections obtained here should be useful as estimates of the importance of "comover" scattering in suppressing heavyquarkonium production, which is of considerable interest in the search for the quark-gluon plasma.

Our results show that the cross section for the dissociation of $J / \psi$ by $\pi$ occurs at a relatively high threshold, and the peak total cross section is about $1 \mathrm{mb}$. In contrast, the cross section for the dissociation of $\psi^{\prime}$ by $\pi$ occurs at a low threshold and the cross section is much larger. We have also evaluated the corresponding cross section for the dissociation by 
$\rho$. This process is exothermic, and has a large total dissociation cross section that diverges at threshold.

We previously noted that our $\pi+J / \psi$ cross section is considerably smaller than the estimate of Ref. [20], although we use a similar approach. There are several differences between the two approaches which lead to this discrepancy. First, Martins et al. assumed that the confining interaction is an attractive Gaussian potential which acts only between quark-antiquark pairs. The neglect of the quark-quark and antiquark-antiquark confining interaction amounts to discarding the transfer diagrams (T1 and T2) for the confining potential. Since we find that the transfer and capture diagram confinement contributions are similar in magnitude but opposite in sign (due to color matrix elements), the confining interaction assumed by Martins et al. did not incorporate this important destructive interference. Second, their use of a Gaussian, rather than the usual linear confining potential, obviously leads to quantitatively different cross sections.

The destructive interference between transfer and capture diagrams with spinindependent forces (color-Coulomb and confinement) has been noted previously. (See, for example, Refs. 33, 34] and references cited in [37].) This interference explains the wellknown spin-spin hyperfine dominance in light hadron scattering in channels such as $I=2$ $\pi \pi$, and the core $N N$ interaction. With heavy quarks, however, the hyperfine interaction contribution is smaller due to the large charm quark mass; for this reason we included the color-Coulomb and confining interactions in our analysis. Our results indicates that the spin-spin, the linear interaction, and the color-Coulomb interactions all give important contributions to the dissociation cross sections.

It is of interest to compare our results of the dissociation cross section with those obtained in the meson exchange model with effective Lagrangians [24 27]. In the effective Lagrangian approach, the dissociation cross section increases with energy, as expected for the $t$-channel exchange of a spin-one particle. For example in Ref. [26 the dissociation cross section is about $30 \mathrm{mb}$ for $\pi+J / \psi \rightarrow D+\bar{D}^{*}$ and about $80 \mathrm{mb}$ for $\pi+\Upsilon \rightarrow B+\bar{B}^{*}$, at $1 \mathrm{GeV}$ above the threshold. These large cross sections continue to increase with increasing energy. In contrast, in our quark models calculation using the Barnes-Swanson model, we find very small cross sections this far above threshold for $\pi+J / \psi \rightarrow D+\bar{D}^{*}$ and $\pi+\Upsilon \rightarrow B+\bar{B}^{*}$. The predicated peak cross section for $\pi+J / \psi \rightarrow D+\bar{D}^{*}$ is about $0.5 \mathrm{mb}$ and occurs at about $0.05 \mathrm{GeV}$ from the threshold. The predicted peak cross section for $\pi+\Upsilon \rightarrow B+\bar{B}^{*}$ is even smaller (about $0.03 \mathrm{mb}$ ), and it occurs at about $0.02 \mathrm{GeV}$ from the threshold. These cross sections decrease rapidly at higher energies. Thus, the large cross sections obtained in the effective Lagrangian approach differ by orders of magnitude from the quark model results obtained here. We believe that the large increase in these dissociation cross sections predicted by the effective Lagrangian meson exchange models at high energies is unrealistic, since the momentum distributions of the boosted final and the initial states have little overlap at high energies.

As the effective Lagrangian approach does not contain information about the internal structure of the interacting hadrons, phenomenological form factors have been introduced to reduce the theoretical cross sections [25 27]. A realistic description of the form factors should incorporate the meson wave functions and the dynamics of the scattering process. Without a derivation of these form factors, one encounters considerable uncertainty, as experimental data on these reaction processes are unavailable. The form factors introduced 
in 25 27] lead to changes of the theoretical cross section at high energies by several orders of magnitude. The results are sensitive both to the assumed coupling strength and to the functional dependence of the form factor. In view of the strong dependence of the theoretical results on the form factor and the coupling constants, a careful determination of these quantities are required in future work.

Although there is no direct experimental measurement of these cross sections to which we can compare our results, the small $\pi+J / \psi$ and the large $\pi+\psi^{\prime}$ dissociation cross section at low kinetic energies obtained here appear qualitatively consistent with earlier results in a microscopic model of $J / \psi$ and $\psi^{\prime}$ suppression in $\mathrm{O}+\mathrm{A}$ and $\mathrm{S}+\mathrm{U}$ collisions [8,9]. Hopefully, future Monte Carlo simulations of the dynamics of charmonium in heavy-ion collisions will lead to a more direct comparison. It is interesting to note that dissociation of $J / \psi$ by $\pi$ and $\rho$ populate different states (for example, $\pi+J / \psi$ does not lead to $D \bar{D}$ in our model but $\rho+J / \psi$ does). It may provide a way to separate these processes by studying the relative production of $D \bar{D}, D^{*} \bar{D}, D \bar{D}^{*}$, and $D^{*} \bar{D}^{*}$, if the open charm background can be subtracted. However, the large ratio of initial open charm to $J / \psi$ production in a nucleon-nucleon collision may make this separation very difficult.

In the future it may be useful to carry out detailed simulations of $J / \psi$ absorption in heavy-ion collisions using the cross sections obtained here. If our cross sections do prove to be reasonably accurate, it will clearly be useful to incorporate them in simulations of hadron processes in relativistic heavy-ion collisions that use the $J / \psi$ suppression as a signature of the quark-gluon plasma, in order to isolate the effects of $J / \psi$ suppression due to its interaction with hadron matter.

\section{ACKNOWLEDGMENTS}

This research was supported by the Division of Nuclear Physics, Department of Energy, under Contract No. DE-AC05-00OR22725 managed by UT-Battelle, LLC. ES acknowledges support from the DOE under grant DE-FG02-00ER41135 and DOE contract DE-AC0584ER40150 under which the Southeastern Universities Research Association operates the Thomas Jefferson National Accelerator Facility. The authors would also like to thank Drs. C. M. Ko, H. Crater, and S. Sorensen for useful discussions.

\section{Appendix A: Tabulation of Bound State Wave Functions}

The wavefunction in reduced momentum $2 \boldsymbol{p}=\boldsymbol{p}_{q}-\boldsymbol{p}_{\bar{q}}$ is represented as a linear combination of Gaussian wave functions with linearly-spaced $\beta^{2}$, of the form

$$
\Phi(2 \boldsymbol{p})=\sum_{n=1}^{N} a_{n} \phi_{n}(2 \boldsymbol{p})=\sum_{n=1}^{N} a_{n}\left(\frac{1}{\pi n \beta^{2}}\right)^{3 / 4} \frac{1}{\left(2 n \beta^{2}\right)^{l / 2}}(2 p)^{l} \sqrt{\frac{4 \pi}{(2 l+1) ! !}} Y_{l m}(\hat{\boldsymbol{p}}) \exp \left\{-\frac{(2 \boldsymbol{p})^{2}}{8 n \beta^{2}}\right\}
$$

where $\Phi(\mathbf{2 p})$ and $\phi_{n}(2 \boldsymbol{p})$ are normalized according to Eqs. (32) and (30),

$$
\int d \boldsymbol{p}|\Phi(\mathbf{2} \boldsymbol{p})|^{2}=\int d \boldsymbol{p}\left|\phi_{n}(\mathbf{2} \boldsymbol{p})\right|^{2}=1 .
$$

The coefficients $\left\{a_{n}\right\}$ for each meson in an $N=6$ basis, with a different $\beta$ for each meson, are listed in the following table. 


\begin{tabular}{|c|c|c|c|c|c|c|c|c|c|}
\hline Meson & $\begin{array}{c}M(\exp ) \\
(\mathrm{GeV})\end{array}$ & $\begin{array}{l}M(\mathrm{th}) \\
(\mathrm{GeV}) \\
\end{array}$ & $\begin{array}{c}\beta \\
(\mathrm{GeV}) \\
\end{array}$ & $a_{1}$ & $a_{2}$ & $a_{3}$ & $a_{4}$ & $a_{5}$ & $a_{6}$ \\
\hline$\pi$ & 0.140 & 0.140 & 0.380 & 8288 & 0.5178 & .2294 & 4.0001 & 5.8837 & 2.9139 \\
\hline $\bar{K}$ & 0.494 & 0.495 & 0.440 & 1.4258 & -2.9104 & 6.6580 & -7.6222 & 4.1972 & -0.6622 \\
\hline$K^{*}$ & 0.892 & 0.904 & 0.440 & 2.6690 & -7.7381 & 18.5854 & -25.2611 & 17.6588 & -4.9261 \\
\hline$\rho$ & 0.770 & 0.774 & 0.380 & 2.5214 & -6.9921 & 16.7985 & $\mid-22.9186$ & 16.1163 & -4.5409 \\
\hline$\overline{\phi(1 s)}$ & 1.020 & 0.992 & .380 & 1.4078 & \begin{tabular}{|l|}
-2.2292 \\
\end{tabular} & 5.2488 & 976 & 4.0718 & -0.9727 \\
\hline$b_{1}$ & 1.235 & 1.330 & 0.380 & 2.2568 & -5.4759 & 12.6496 & -17.1515 & 12.1528 & -3.4443 \\
\hline$a_{1}$ & 1.260 & 1.353 & 0.380 & 2.3362 & -5.7733 & 13.3524 & -18.2221 & 13.0172 & -3.7466 \\
\hline$\phi(2 s)$ & 1.686 & 1.870 & 0.380 & 5.7964 & -24.6635 & 58.1365 & $\mid-79.9357$ & 56.8725 & -16.2428 \\
\hline$D$ & 69 & 13 & 40 & 1.8275 & -4.2160 & 10.0225 & -13.0384 & 8.6764 & -2.2285 \\
\hline$D^{*}$ & 2.010 & 1.998 & 0.440 & 2.1630 & -5.4765 & 13.0711 & -17.5068 & 12.0520 & -3.2893 \\
\hline$D_{s}$ & 1.969 & 2.000 & 0.440 & 1.0701 & -1.1418 & 2.4522 & -1.9688 & 0.3196 & 0.3292 \\
\hline$D_{s}^{*}$ & 2.112 & 2.072 & 0.440 & 1.3267 & -1.9616 & 4.5086 & -5.2172 & 2.9478 & -0.5646 \\
\hline$D_{1}\left({ }^{1} P_{1}\right)$ & 2.422 & 2.506 & 0.440 & 2.2042 & -5.2226 & 12.0872 & -16.4595 & 11.7325 & -3.3595 \\
\hline$D_{2}\left({ }^{3} P_{2}\right)$ & 2.460 & 2.514 & 0.440 & 2.2344 & -5.3296 & 12.3210 & -16.7896 & 11.9756 & -3.4375 \\
\hline$\eta_{c}$ & 2.979 & 3.033 & 0.560 & 0.9461 & -0.6474 & 1.0666 & 0.3614 & -1.6509 & 0.9868 \\
\hline$J / \psi$ & 3.097 & 3.069 & 0.560 & 1.0786 & -1.0517 & 2.0729 & 89 & -0.3804 & 0.5646 \\
\hline$h_{c}$ & 3.570 & 3.462 & 0.560 & 1.6312 & 87 & 68 & -9. & 51 & -1.8163 \\
\hline$\chi_{c}$ & 3.525 & 3.466 & 0.560 & 1.6587 & -2.9420 & 6.8805 & -9.3051 & 6.5918 & -1.8698 \\
\hline$\psi^{\prime}$ & 3.686 & 3.693 & 0.560 & 5.5237 & -22.5889 & 53.5145 & -74.6754 & 53.9666 & -15.7222 \\
\hline$B$ & 5.279 & 5.322 & 0.500 & 2.4905 & -7.0584 & 17.0138 & -23.0809 & 16.1128 & -4.4690 \\
\hline$B^{*}$ & 5.324 & 5.342 & 0.500 & \begin{tabular}{|l|}
2.5806 \\
\end{tabular} & \begin{tabular}{|l|}
-7.4190 \\
\end{tabular} & 17.8694 & -24.2889 & 16.9859 & -4.7275 \\
\hline$\overline{B_{s}}$ & 5.369 & 5.379 & 0.500 & \begin{tabular}{|l|}
1.6289 \\
\end{tabular} & -3.2614 & 7.6922 & -9.8055 & 6.3702 & -1.5866 \\
\hline$B_{s}^{*}$ & 5.416 & 5.396 & 0.500 & \begin{tabular}{|l|}
1.7111 \\
\end{tabular} & -3.5483 & 8.3718 & -10.7991 & 7.1136 & -1.8177 \\
\hline$\Upsilon(1 s)$ & 9.460 & 9.495 & 0.660 & 0.1364 & 2.0441 & -6.7818 & 14.2875 & -13.9803 & 5.3693 \\
\hline$\chi_{b}(1 p)$ & 9.899 & 9.830 & 0.660 & \begin{tabular}{|l|}
0.7416 \\
\end{tabular} & 0.1481 & 0.2178 & -0.0196 & -0.1923 & 0.1587 \\
\hline$\Upsilon(2 s)$ & 10.020 & 9.944 & 0.660 & -3.6422 & 10.7655 & -25.3407 & 38.7395 & -30.5647 & 9.8544 \\
\hline$\chi_{b}(2 p)$ & 10.260 & 10.166 & 0.660 & 3.2645 & -10.1170 & 20.7960 & -28.2373 & 20.2666 & -5.9472 \\
\hline
\end{tabular}




\section{REFERENCES}

[1] Quark Matter '99, Proceedings of the 14th International Conference on UltraRelativistic Nucleus-Nucleus Collisions, Editors L. Riccati, M. Masera, and E. Vercellin, Nuclear Physics A, Volume A661 (1999).

[2] C. Y. Wong, Introduction to High-Energy Heavy-Ion Collisions, World Scientific Publishing Company, 1994.

[3] U. Heinz and M. Jacobs, nucl-th/0002042.

[4] C. Y. Wong, Nucl. Phys. A681, 22c (2001).

[5] T. Matsui and H. Satz, Phys. Lett. B178, 416 (1986).

[6] M. Gonin, NA50 Collaboration, Nucl. Phys. A610, 404c (1996).

[7] A. Romana et al., NA50 Collaboration, in Proceedings of the XXXIII Recontres de Moriond, Les Arcs, France, 21-28 March, 1998.

[8] C. Y. Wong, Nucl. Phys. A610, 434c (1996); Nucl.Phys. A630, 487 (1998).

[9] C. Y. Wong, hep-ph/9809497, in proceedings of Workshop on Charmonium Production in Relativistic Nuclear Collisions, (INT, Seattle, May 18-22, 1998).

[10] D. Kharzeev, Nucl. Phys. A610, 418c (1996); Nucl. Phys. A638, 279c (1998).

[11] J.-P. Blazoit and J.-Y. Ollitrault, Nucl. Phys. A610, 452c (1996).

[12] A. Capella, A. Kaidalov, A. K. Akil, and C. Gerschel, Phys. Lett. B393, 431 (1997).

[13] W. Cassing and C. M. Ko, Phys. Lett. B396, 39 (1997); W. Cassing, E. L. Bratkovskaya, Nucl. Phys. A623, 570 (1997).

[14] R. Vogt, Phys. Lett. B430 15 (1998).

[15] M. Nardi and H. Satz, Phys. Lett. B442 14 (1998).

[16] Bin Zhang, C.M. Ko, Bao-An Li, Ziwei Lin, and Ben-Hao Sa, Phys.Rev. C62, 054905 (2000).

[17] D.B. Blaschke, G. R. G. Burau, M. I. Ivanov, Yu. L. Kalinovsky, and P. C. Tandy, Phys. Lett. B506 297 92001).

[18] D. Kharzeev and H. Satz, Phys. Lett. B334, 155 (1994).

[19] D. Kharzeev, H. Satz, A. Syamtomov, and G. Zinovjev, Phys. Lett. B389, 595 (1996).

[20] K. Martins, D. Blaschke, and E. Quack, Phys. Rev. C51, 2723 (1995).

[21] C. Y. Wong, E. S. Swanson, and T. Barnes, Phys. Rev. C62, 045201, 2000.

[22] C. Y. Wong, E. S. Swanson, T. Barnes, in Proceedings of the Hirschegg 2000 Workshop on Hadrons in Dense Matter (Hirschegg, Austria, 16-22 January 2000); nucl-th/000203.

[23] T.Barnes, E.S.Swanson, and C.Y.Wong, Proceedings of International Workshop on Heavy Quark Physics 5 (Dubna, 6-8 April 2000), nucl-th/0006012.

[24] S. G. Matinyan and B. Müller, Phys. Rev. C58, 2994 (1998).

[25] K. L. Haglin, Phys. Rev. C61, 031912 (2000); K. L. Haglin and C. Gale, Phys. Rev. C63 065201 (2001).

[26] Z. W. Lin and C. M. Ko, Phys. Rev. C62, 034903 (2000).

[27] Yongseok Oh, Taesoo Song, and Su Houng Lee, Phys. Rev. C63, 034901 (2001).

[28] A. Sibirtsev, K. Tsushima, and A. W. Thomas, Phys. Rev. C63, 044906 (2001).

[29] M. Peskin, Nucl. Phy. B156, 365 (1979).

[30] G. Bhanot and M. Peskin, Nucl. Phy. B156, 391 (1979).

[31] L. Antoniazzi et al., Phys. Rev. Lett. 70, 383 (1993).

[32] Ziwei Lin and C. M. Ko, Phys. Lett. B503, 1042001.

[33] T. Barnes and E. S. Swanson, Phys. Rev. D46, 131 (1992). 
[34] E. S. Swanson, Ann. Phys. (N.Y.) 220, 73 (1992).

[35] T. Barnes, E. S. Swanson, and J. Weinstein, Phys. Rev. D46, 4868 (1992).

[36] T. Barnes and E. S. Swanson, Phys. Rev. C49, 1166 (1994).

[37] T. Barnes, S. Capstick, M. D. Kovarik, and E. S. Swanson, Phys. Rev. C48, 539 (1993).

[38] T. Barnes, N. Black, D. J. Dean, and E. S. Swanson, Phy. Rev. C60, 045202 (1999).

[39] L. I. Schiff, Quantum Mechanics (McGraw-Hill, New York, 1968), pp.384-387.

[40] A. de-Shalit and I. Talmi, Nuclear Shell Model, Academic Press, 1963.

[41] W. Hoogland et al., Nucl. Phys. B126, 109 (1977).

[42] C.Y. Wong and H. Crater, Phys. Rev. C63, 044907 (2001).

[43] T. Barnes, E. Swanson, and C.Y. Wong, to be published. 